\title{
Magnetically suspended flywheel in gimbal mount - Nonlinear modelling and
} simulation

Dagnæs-Hansen, Nikolaj A.; Santos, IImar F.

Published in:

Journal of Sound and Vibration

Link to article, DOI:

10.1016/j.jsv.2018.06.033

Publication date:

2018

Document Version

Peer reviewed version

Link back to DTU Orbit

Citation (APA):

Dagnæs-Hansen, N. A., \& Santos, I. F. (2018). Magnetically suspended flywheel in gimbal mount - Nonlinear modelling and simulation. Journal of Sound and Vibration, 432, 327-350.

https://doi.org/10.1016/j.jsv.2018.06.033

\section{General rights}

Copyright and moral rights for the publications made accessible in the public portal are retained by the authors and/or other copyright owners and it is a condition of accessing publications that users recognise and abide by the legal requirements associated with these rights.

- Users may download and print one copy of any publication from the public portal for the purpose of private study or research.

- You may not further distribute the material or use it for any profit-making activity or commercial gain

- You may freely distribute the URL identifying the publication in the public portal

If you believe that this document breaches copyright please contact us providing details, and we will remove access to the work immediately and investigate your claim. 


\title{
Magnetically Suspended Flywheel in Gimbal Mount - Nonlinear Modelling and Simulation
}

\author{
Nikolaj A. Dagnaes-Hansen, Ilmar F. Santos* \\ Department of Solid Mechanics, Technical University of Denmark, Kgs. Lyngby, Denmark
}

\begin{abstract}
Flyweel energy storage systems (FESSs) with active and passive magnetic bearings are generating interest due to their increasing energy-storing potential caused by advances in motor, bearing and fibre-composite technology. Magnetically suspended FESS are used commercially on static foundations while vehicle applications pose challenges due to manoeuvring, impacts, and other outer perturbations and have thus only seen successful experimental application in a few research projects where the FESS has been mounted in a passive gimbal to avoid gyroscopic forces. Although experimentally implemented, a mathematical model is still missing that determines motions and forces when the FESS is suspended in magnetic bearings, gimbalmounted, and subject to outer perturbations. This work thoroughly describes how to set up a mathematical model that couples the multi-body dynamics of a flywheel rotor, housing, and gimbal-mount with the magnetic forces of the bearings. The model is used to simulate the behaviour of a FESS with and without gimbal and subject to various perturbations. The results demonstrate how the gimbal mount effectively removes gyroscopic forces but introduces other potential challenges such as large rotor and housing displacements due to dynamical interactions between the rotor, active magnetic bearings, housing, and gimbal.
\end{abstract}

Keywords: Flywheel Energy Storage; Magnetic Bearings; Multi-body dynamics; Electromagnetism; Rotordynamics; Multiphysics.

\section{Introduction}

This work is motivated by the increasing interest in flywheel based energy storage systems (FESS) where kinetic energy is stored by rotating a flywheel. The amount of stored energy of such a system is quadratically increasing with rotational speed. In order to optimise energy density, the rotational speed is increased to 5 magnitudes where magnetic bearings become paramount. The magnetic suspension can consist of active magnet bearings or a combination of active and passive magnetic bearings. It can function in a vacuum, it has low friction and wear, and can compensate rotor unbalance giving it superiority over other types of

\footnotetext{
*Corresponding author. Tel.: +45 256269

Email address: ifs@mek.dtu.dk (Ilmar F. Santos)
} 


\section{Symbol Meaning}

\section{Units}

$\alpha$

First gimbal rotation

$\mathrm{rad}$

$\beta \quad$ Second gimbal rotation

$\mathrm{rad}$

$\Delta \quad$ Radial displacement of rotor

$\frac{\pi}{2}$-latitude of vehicle with FESS

$\mathrm{rad}$

First rotor tilt angle

$\operatorname{rad}$

Second rotor tilt angle

$\mathrm{rad}$

Vehicle pitch angle

rad

$\Theta \quad$ Circumferential coordinate

$\mathrm{rad}$

Permeability in vacuum

$\mathrm{H} / \mathrm{m}$

Friction coefficient of bearing

$\mathrm{m}^{2} \mathrm{~N} / \mathrm{s}$

$\tau$

Torque

$\mathrm{Nm}$

Vehicle roll angle

$\mathrm{rad}$

Vehicle yaw angle

$\mathrm{rad}$

Rotational speed

$\mathrm{rad} / \mathrm{s}$

Length from rotor CM to AMB A m

\section{$B_{i}$}

$B_{i}$

Body attached frame number $i$

$B$

Magnetic field density magnitude

-

\section{$\mathrm{T}$}

Length from rotor CM to AMB B m

c Length from rotor CM to sensor A m

$d \quad$ Length from rotor CM to sensor B m

E Electric field magnitude $\quad \mathrm{V} / \mathrm{m}$

$e \quad$ Length from rotor CM to PMB m

G Gimbal outer diameter m

$g \quad$ Acceleration of gravity $\mathrm{m} / \mathrm{s}^{2}$

$H \quad$ Magnetic intensity A

h Gimbal inner diameter m

$\begin{array}{lll}h_{0} & \text { PMB air gap }\end{array}$

I Inertial frame -

$I_{X_{p}} \quad$ Polar moment of inertia of $\mathrm{X} \quad \mathrm{kg} \mathrm{m}^{2}$

$I_{X_{t}} \quad$ Transversal moment of inertia of $\mathrm{X} \quad \mathrm{kg} \mathrm{m}^{2}$

$i \quad$ Current A

$K_{C} \quad$ Amplifier Gain V/A

$K_{i} \quad$ Integral Gain $1 / \mathrm{s}$

$k_{i} \quad$ Force-current factor N/A 


$\begin{array}{llc}\text { Symbol } & \text { Meaning } & \text { Units } \\ K_{p} & \text { Proportional Gain } & \mathrm{A} / \mathrm{m} \\ k_{r} & \text { PMB radial force-displacement factor } & \mathrm{N} / \mathrm{m} \\ k_{s} & \text { Force-displacement factor } & \mathrm{N} / \mathrm{m} \\ k_{u} & \text { Motion induced voltage coefficient } & \mathrm{V} /(\mathrm{m} / \mathrm{s}) \\ k_{z} & \text { PMB axial force-displacement factor } & \mathrm{N} / \mathrm{m} \\ L & \text { Inductance } & \mathrm{H} \\ l & \text { Length from gimbal's CM to housing's CM } & \mathrm{m} \\ l_{u} & \text { Length from rotor CM to point of attack of unbalance force } & \mathrm{m} \\ M & \text { Magnetic Moment } & \mathrm{A} / \mathrm{m} \\ m & \text { Mass } & \mathrm{kg} \\ n & \text { Number of coil windings } & - \\ n_{r} & \text { Number of current conductors radially } & - \\ n_{z} & \text { Number of current conductors axially } & - \\ r & \text { Radius } & \mathrm{m} \\ s & \text { Air gap } & \mathrm{m} \\ T_{d} & \text { Derivative time gain } & \mathrm{s} \\ t & \text { Time } & \mathrm{t} \\ u & \text { Unbalance amplitude } & \mathrm{g} \cdot \mathrm{mm} \\ w & \text { Permanent magnet height } & \mathrm{m} \\ w_{c} & \text { Cut-off frequency } & \mathrm{m} \\ x_{0} & \text { Nominal AMB air gap } & \mathrm{m} \\ z_{0} & \text { Nominal distance rotor to housing CM } & \end{array}$

\section{Abbreviation Meaning}

AMB

Active Magnetic Bearing

FESS

Flywheel Energy Storage System

KERS

Kinetic Energy Recovery System

PMB

Permanent Magnet Bearing

RPM

Rounds Per Minute

UPS

Uninterruptible Power Supply 
bearings in high speed FESS applications. Currently, FESS with energy densities up to $80 \mathrm{Wh} / \mathrm{kg}$ have been manufactured [1. In comparison, modern lithium-ion batteries can contain up to approximately 180 $\mathrm{Wh} / \mathrm{kg}$ [2]. However, FESS offer advantages over electrochemical batteries such as long life expectancy more than 20 years - no hazardous materials, no toxic or explosive gas emissions, accurate diagnostics and monitoring, and no fire hazard [3. This has made them an interesting battery alternative and commercialised FESS are now available from different suppliers [4 [5] [6] [3. If the rotational speed is further increased and more advanced high strength composite materials are utilised, it is predicted, that FESS energy density will reach more than $2000 \mathrm{Wh} / \mathrm{kg}$ [7]. In [8], a list of 28 different research groups and 27 different manufacturers of flywheels is presented. Interest in the topic remains increasing and at least three reviews have been published in internationally acknowledged journals in 2017 alone [9] [10] [11. The applications include both stationary systems such as uninterruptible power supplies (UPS) or storage for renewable energies (solar and wind), as well as mobile systems such as vehicular, marine, and aerospace applications. For mobile systems however, there is one property that is predominant no matter the application: gyroscopics. The sole purpose of a FESS is to store energy as angular momentum and this means that the flywheel will strongly resist rotations and the system will reciprocate large gyroscopic forces when subject to tilt motions. This can be beneficial, for example in satellite attitude control systems which are based on the principle of exchanging angular momentum [12] 13] [14]. In most cases, however, the large gyroscopic forces are unwanted and difficult to handle for active magnetic bearings. Consequently, vehicular and naval FESSs typically have low energy density and use a suspension made of conventional bearings such as ball bearings. This is the case in kinetic energy recovery systems (KERS) in motor sports [16 [8] [17. Here the magnitudes of velocities and accelerations are too large and the given available space is too compact, for active magnetic bearings to be suitable.

However magnetic suspension is needed in order to increase rotational speeds and decrease friction and wear if mobile FESS is to compete with other types of batteries on energy density, efficiency, and lifetime. This requires an assessment of the dynamic consequences of introducing magnetic suspension in a mobile FESS. The dynamics of magnetic bearings interacting with bodies such as rotor, housing, and other suspension parts as well as the accelerations of the vehicle/vessel from e.g. manoeuvring or outer perturbations are crucial for the whole system design. Therefore, the problem at hand consists of how to model magnetically suspended high-inertia flywheels on moving foundations such that important dynamics are successfully captured and the system can be properly designed.

The articles [18, [19], and 20] provide comprehensive overviews of previous literature concerning magnetically suspended rotors subject to base motion. Here, various control schemes are used to handle gyroscopic forces. Looking specifically into magnetically suspended FESS rather than rotors in general, we see a general trend of using gimbals to compensate gyroscopic forces. The Center of Electromechanics at University of Texas have been specialising in FESS for bus application. They are among the first to use AMBs for mobile 
FESS and one of their first articles quantifies bearing loads taking into account vehicle shock, vibration, manoeuvring, and gyrodynamics 21]. They conclude that the flywheel spinning axis must be oriented vertically and that a passive gimbal mount is necessary to avoid large gyroscopic forces. Their original contribution is using real acceleration data from transit buses as their outer perturbations. They also advise that the gimbal mount must incorporate springs and dampers in order to restrain precession movements. They present novel experimental work of a gimballed FESS with magnetic suspension subject to external perturbations in 22]. Their model only comprises three degrees of freedom governing axial motion. Nonetheless, the model captures a small part of the dynamics essential for design considerations. They place the flywheel center of mass below the gimbal pivotal axes giving the flywheel a natural vertical home position (pendulum type spring). In [23], they present endurance performance testing and they extend their tests beyond bus motions using a terrain simulator. Another successful integration of magnetically suspended FESS in a vehicle is found in 24] 25]. A reconfigured golf cart is used to experimentally test a FESS spinning up to 18000 RPM and subject to accelerations from the moving cart. Here the FESS is mounted vertically in an active gimbal. The AMBs are controlled using adaptive control. For marine application a few conceptual designs have been developed by commercial suppliers. In [26, the company Calnetix is presenting a suggested FESS for naval applications. The starting point of the design is the above mentioned FESS for bus application developed at the University of Texas. Since this concept has already proven functional in a mobile framework, they put little focus on ship movements and their consequences. The company Beacon Power together with the Naval Surface Warfare Center presents in [27] a discussion of technical challenges including dynamic shipboard environment and flywheel shock tolerances. Instead of a gimbal mount, they propose the flywheel spin axis to be mounted parallel to the ship's keel axis. This way, precession torques caused by ship rolling are mitigated.

\section{Original contribution of this work}

As seen from the literature, a strong focus has been on the gyrodynamic forces and how to avoid them for mobile FESS application; for example, using a gimbal mount as in [21] 22] [23] 24] 25]. However, the dynamics of a passive gimbal mounted rotor with AMBs have, to the best knowledge of the authors, never been assessed before. This work will examine the interacting dynamics of rotor, magnetic bearings, housing, and a passive gimbal. The original contribution will consist of coupling a multi-body model of an externally perturbated gimballed flywheel with the magnetic forces from active and passive magnetic bearings. The gimbal and the rotor housing are free to rotate and are thus dynamically interacting with the rotor-bearing system. The inertia of gimbal and housing are included and their effects carefully investigated. Nonlinear permanent magnetic forces and active magnetic forces including control algorithm are incorporated in the model. To demonstrate the application of the model, a parametric study of the dynamics when varying dimensions of rotor and housing is conducted. 


\section{Approach}

The equations of motion are derived using the Newton-Euler method [28, 29] [30] [31 following the same procedure as in [32] 33. The forces from the active magnetic bearings are derived from first principles as in e.g. 34. The procedure used here can be used for most types of AMBs although the outcome will vary with type of bearings. The control and signal conditioning of the AMBs is sought as simple as possible while still accurately representing what is most commonly implemented in practice. Some magnetically suspended flywheels also use permanent magnet bearings. These often consist of ring-type permanent magnets such as in [35] 36] 37 38, 39, 40. Therefore, this work will also give an example of how to estimate forces from a simple ring-type permanent magnet bearing. A derivation from first principles is unfortunately not available. Instead numerical methods will be used similar to the method used in 41, 42. Again, the procedure can be used for most types of permanent magnet bearings although the outcome will vary with type of bearing. Finally when using a gimbal mount, the system starts to alarmingly resemble a gyrocompass. Therefore,

the effect of the gyroscopic interactions with the earth and the flywheels spin will also be investigated.

\section{Mathematical Modelling}

\section{Multi-body Model}

The final outcome of the multi-body modelling will be the equations of motion, Eq. (18)-(23). In order to obtain these equations, reference systems, position vectors and kinematics relations first have to be defined and formulated.

\section{(a) Definition of the inertial and Moving Reference Systems}

Nine moving reference frames $B_{0}, B_{0^{\prime}}, B_{1}$ to $B_{7}$ and one inertial $I\left(X_{I}, Y_{I}, Z_{I}\right)$ frame are defined as seen in Fig. 1 and Fig. 2

Frame $B_{0}$ is attached to the earth's center of mass $(\mathrm{CM})$. Frame $B_{0^{\prime}}$ is attached to the earth's surface. The frames $B_{1}$ to $B_{3}$ are attached to the vehicle's CM, frame $B_{4}$ is attached to the gimbal's CM, frame $B_{5}$ is attached to the housing's CM, and frames $B_{6}$ and $B_{7}$ are attached to the rotor's CM. Frame $B_{0}\left(X_{0}, Y_{0}, Z_{0}\right)$ is obtained by rotating $I$ the angle $\left(\Omega_{\text {earth }} t\right)$ around the $Z_{I}$-axis (earth's spin). Consequently, a vector that is represented in the inertial frame as ${ }_{I} \mathbf{r}$, will be expressed in $B_{0}$ by $B_{0} \mathbf{r}$ as

$$
B_{0} \mathbf{r}={ }_{B_{0}} \mathbf{T}_{I I} \mathbf{r}, \quad B_{0} \mathbf{T}_{I}=\left[\begin{array}{ccc}
\cos \left(\Omega_{\text {earth }} t\right) & \sin \left(\Omega_{\text {earth }} t\right) & 0 \\
-\sin \left(\Omega_{\text {earth }} t\right) & \cos \left(\Omega_{\text {earth }} t\right) & 0 \\
0 & 0 & 1
\end{array}\right]
$$



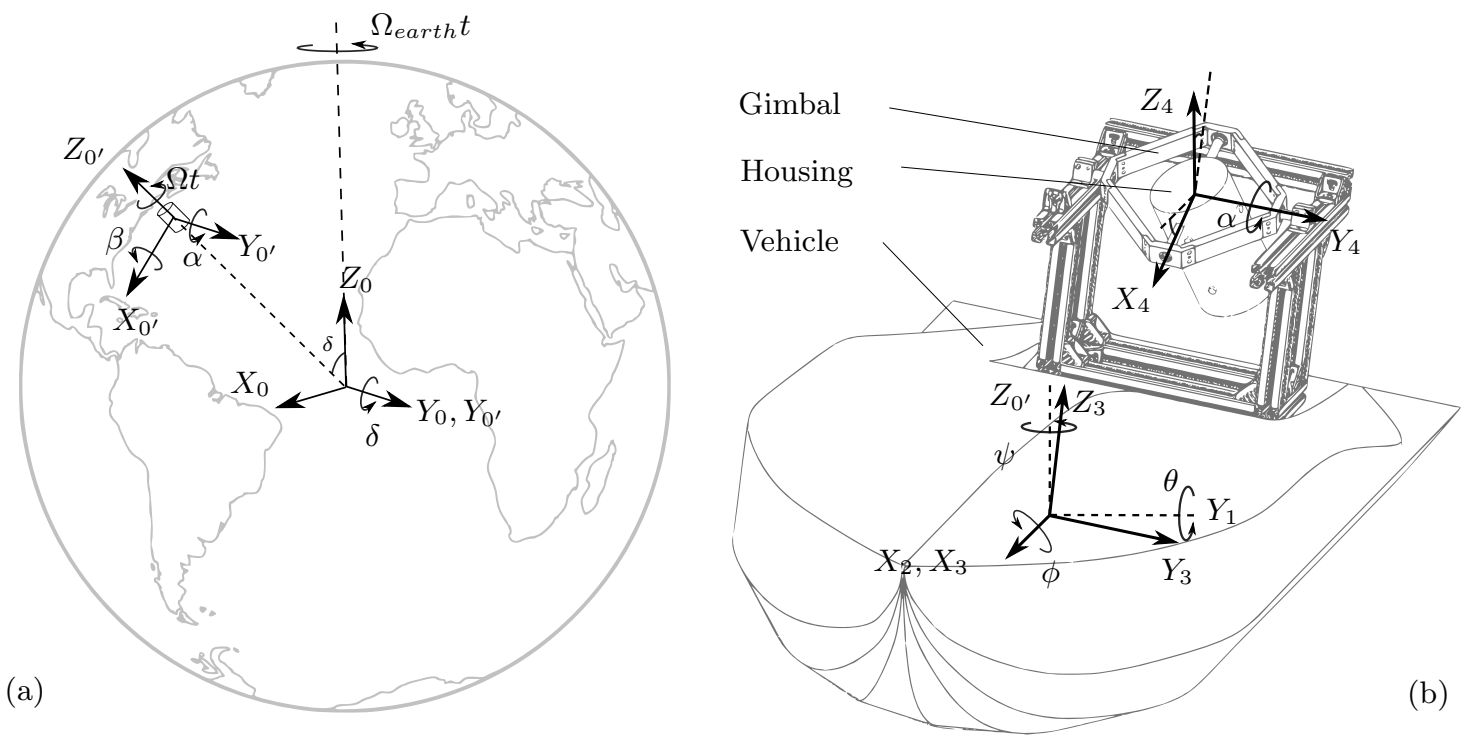

Figure 1: (a): Frame $B_{0}$ is attached to earth $\mathrm{CM}$ with $Z_{0}$ following earth's spinning axis. Frame $B_{0^{\prime}}$ is attached to the surface of the earth at the location of the flywheel-powered vehicle. Consequently $X_{0^{\prime}}$ is pointing south. (b): Vehicle with gimballed flywheel energy storage system on board. Frames $B_{1}$ to $B_{3}$ are used to describe yaw $\psi$, pitch $\theta$, and roll $\phi$ motion.
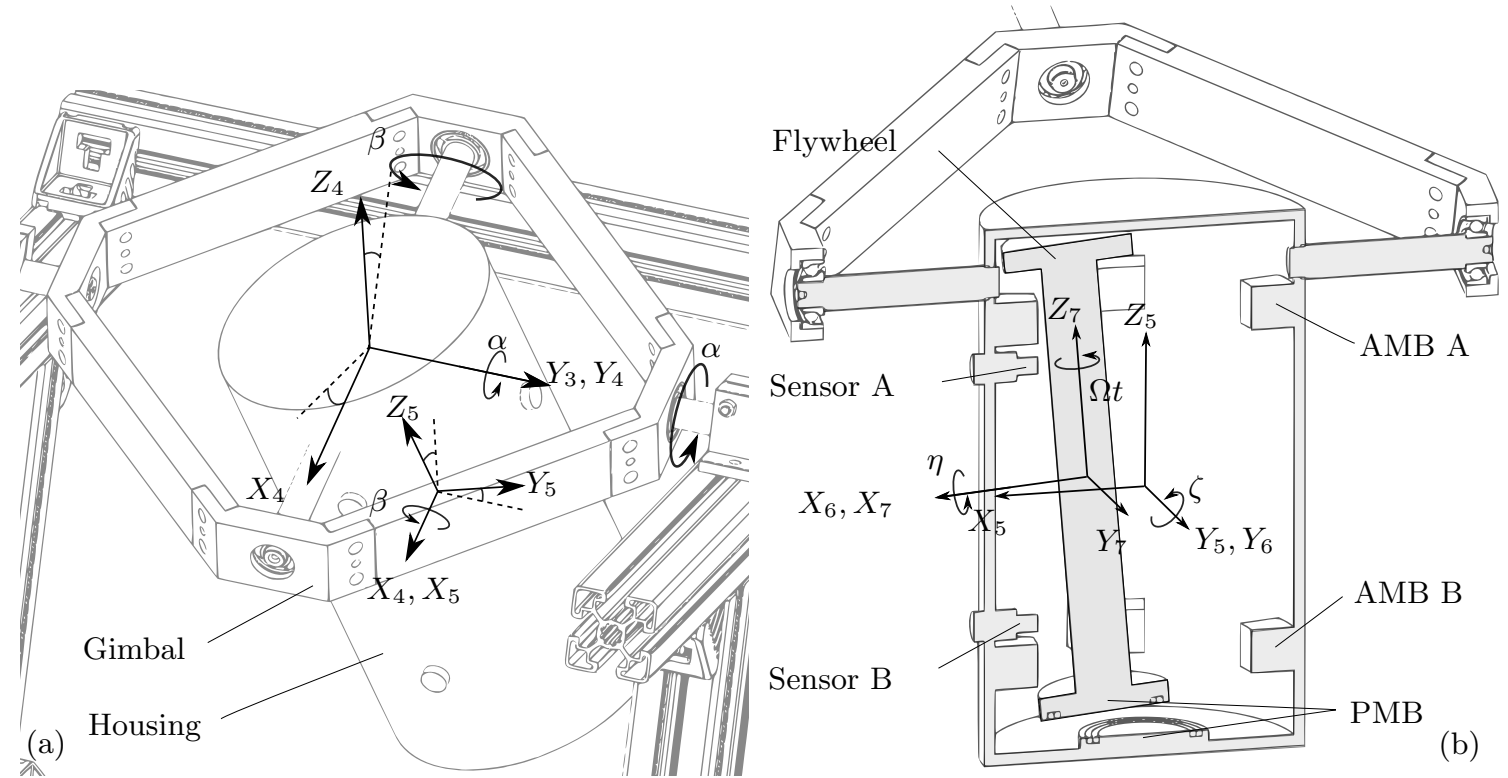

Figure 2: (a): Close-up on flywheel energy storage system with frames attached to gimbal and flywheel housing. (b): Crosssectional view of flywheel housing and flywheel rotor. The magnetic suspension consists of two radial active magnetic bearings (AMBs) and one axial permanent magnetic bearing (PMB). 
in $B_{0}$ coordinates. The eight remaining moving reference frames are defined by

$$
\begin{aligned}
& B_{0^{\prime}} \mathbf{T}_{B_{0}}=\left[\begin{array}{ccc}
\cos \delta & 0 & -\sin \delta \\
0 & 1 & 0 \\
\sin \delta & 0 & \cos \delta
\end{array}\right] B_{1} \mathbf{T}_{B_{0^{\prime}}}=\left[\begin{array}{ccc}
\cos \psi(t) & \sin \psi(t) & 0 \\
-\sin \psi(t) & \cos \psi(t) & 0 \\
0 & 0 & 1
\end{array}\right] \\
& B_{2} \mathbf{T}_{B_{1}}=\left[\begin{array}{ccc}
\cos \theta(t) & 0 & -\sin \theta(t) \\
0 & 1 & 0 \\
\sin \theta(t) & 0 & \cos \theta(t)
\end{array}\right] B_{B_{3}} \mathbf{T}_{B_{2}}=\left[\begin{array}{ccc}
1 & 0 & 0 \\
0 & \cos \phi(t) & \sin \phi(t) \\
0 & -\sin \phi(t) & \cos \phi(t)
\end{array}\right] \\
& B_{4} \mathbf{T}_{B_{3}}=\left[\begin{array}{ccc}
\cos \alpha(t) & 0 & -\sin \alpha(t) \\
0 & 1 & 0 \\
\sin \alpha(t) & 0 & \cos \alpha(t)
\end{array}\right] B_{B_{5}} \mathbf{T}_{B_{4}}=\left[\begin{array}{ccc}
\frac{\sqrt{2}}{2} & \frac{\sqrt{2} \cos \beta(t)}{2} & \frac{\sqrt{2} \sin \beta(t)}{2} \\
-\frac{\sqrt{2}}{2} & \frac{\sqrt{2} \cos \beta(t)}{2} & \frac{\sqrt{2} \sin \beta(t)}{2} \\
0 & -\sin \beta(t) & \cos \beta(t)
\end{array}\right] \\
& B_{6} \mathbf{T}_{B_{5}}=\left[\begin{array}{ccc}
\cos \zeta(t) & 0 & -\sin \zeta(t) \\
0 & 1 & 0 \\
\sin \zeta(t) & 0 & \cos \zeta(t)
\end{array}\right] B_{B_{7}} \mathbf{T}_{B_{6}}=\left[\begin{array}{ccc}
1 & 0 & 0 \\
0 & \cos \eta(t) & \sin \eta(t) \\
0 & -\sin \eta(t) & \cos \eta(t)
\end{array}\right]
\end{aligned}
$$

such that

$$
{ }_{B_{7}} \mathbf{r}={ }_{B_{7}} \mathbf{T}_{I I} \mathbf{r}={ }_{B_{7}} \mathbf{T}_{B_{6} B_{6}} \mathbf{T}_{B_{5} B_{5}} \mathbf{T}_{B_{4} B_{4}} \mathbf{T}_{B_{3} B_{3}} \mathbf{T}_{B_{2} B_{2}} \mathbf{T}_{B_{1} B_{1}} \mathbf{T}_{B_{0^{\prime}} B_{0^{\prime}}} \mathbf{T}_{B_{0} B_{0}} \mathbf{T}_{I I} \mathbf{r}
$$

and

$$
{ }_{I} \mathbf{r}={ }_{B_{7}} \mathbf{T}_{I B_{7}}^{\mathrm{T}} \mathbf{r}={ }_{B_{0}} \mathbf{T}_{I B_{0^{\prime}}}^{\mathrm{T}} \mathbf{T}_{B_{0} B_{1}}^{\mathrm{T}} \mathbf{T}_{B_{0^{\prime}} B_{2}}^{\mathrm{T}} \mathbf{T}_{B_{1} B_{3}}^{\mathrm{T}} \mathbf{T}_{B_{2} B_{4}}^{\mathrm{T}} \mathbf{T}_{B_{3} B_{5}}^{\mathrm{T}} \mathbf{T}_{B_{4} B_{6}}^{\mathrm{T}} \mathbf{T}_{B_{5} B_{7}}^{\mathrm{T}} \mathbf{T}_{B_{6} B_{7}}^{\mathrm{T}} \mathbf{r}
$$

Please note that ${ }_{B_{5}} \mathbf{T}_{B_{4}}$ consists of two rotations - one rotation of angle $\beta$ around $X_{4}$ and one rotation of $45^{\circ}$ around $Z_{5}$. The $45^{\circ}$ rotation is not general and is only present such that the mathematical model can

\section{(b) Position Vectors}

The vectors presented in the sections below, are defined using Figs. 3 and 4 Position of the gimballed flywheel on the vehicle (vector from vehicle's CM to gimbal's CM):

$$
{ }_{B_{3}} \mathbf{r}_{3-4}=\left\{\begin{array}{lll}
x_{3-4} & y_{3-4} & z_{3-4}
\end{array}\right\}^{\mathrm{T}} .
$$

Please note that the gimbal's CM is lying on the axis of the gimbal rotation. Vector from gimbal's CM to housing's CM:

$$
B_{5} \mathbf{r}_{4-5}=\left\{\begin{array}{lll}
0 & 0 & -l
\end{array}\right\}^{\mathrm{T}} .
$$

Position of rotor's CM relative to housing's CM:

$$
{ }_{B_{5}} \mathbf{r}_{5-7}=\{x(t) y(t) z(t)\}^{\mathrm{T}} .
$$


Vectors from rotor's CM to point of attack of magnetic forces:

$$
\begin{array}{ll}
B_{7} \mathbf{r}_{a}=\left\{\begin{array}{lll}
0 & 0 & \left(a^{\prime}-z^{\prime}\right)
\end{array}\right\}^{\mathrm{T}} & B_{7} \mathbf{r}_{b}=\left\{\begin{array}{ll}
0 & 0-\left(b^{\prime}+z^{\prime}\right)
\end{array}\right\}^{\mathrm{T}} \\
{ }_{B_{7}} \mathbf{r}_{e}=\left\{\begin{array}{lll}
0 & 0 & -\left(e^{\prime}+z^{\prime}\right)
\end{array}\right\}^{\mathrm{T}} &
\end{array}
$$

where $a^{\prime}, b^{\prime}, e^{\prime}$, and $z^{\prime}$ are distances along the $Z_{7}$-axis that when projected onto the $Z_{5}$-axis become $a, b, e$, and $z$, e.g.: $a^{\prime}=\frac{a}{\cos \zeta \cos \eta}$.

Vectors from gimbal CM to point of attack of gimbal bearing forces:

$$
\begin{array}{ll}
B_{4} \mathbf{l}_{h_{1}}=\{0-h 0\}^{\mathrm{T}} & B_{4} \mathbf{l}_{h_{2}}=\left\{\begin{array}{lll}
0 & h & 0
\end{array}\right\}^{\mathrm{T}} \\
B_{4} \mathbf{l}_{g_{1}}=\{0-G 0\}^{\mathrm{T}} & B_{4} \mathbf{l}_{g_{2}}=\left\{\begin{array}{lll}
0 & G & 0
\end{array}\right\}^{\mathrm{T}}
\end{array}
$$

Vector from rotor CM to point of attack of rotor unbalance force:

$$
B_{7} \mathbf{r}_{u}=\left\{\begin{array}{lll}
0 & 0 & l_{u}
\end{array}\right\}^{\mathrm{T}}
$$

\section{(c) Kinematic Relations}

Angular velocities of each moving reference frame relative to its preceding frame:

$$
\begin{aligned}
B_{0} \boldsymbol{\Omega}_{\text {earth }} & =\left\{\begin{array}{lll}
0 & 0 & \Omega_{\text {earth }}
\end{array}\right\}^{\mathrm{T}} \\
B_{1} \dot{\boldsymbol{\psi}} & =\left\{\begin{array}{lll}
0 & 0 & \dot{\psi}(t)
\end{array}\right\}^{\mathrm{T}} \\
B_{3} \dot{\boldsymbol{\phi}} & =\left\{\begin{array}{lll}
\dot{\phi}(t) & 0 & 0
\end{array}\right\}^{\mathrm{T}} \\
B_{5} \dot{\boldsymbol{\beta}} & =\left\{\begin{array}{lll}
\frac{\sqrt{2}}{2} \dot{\beta}(t)-\frac{\sqrt{2}}{2} \dot{\beta}(t) & 0
\end{array}\right\}^{\mathrm{T}} \\
B_{7} \dot{\boldsymbol{\eta}} & =\left\{\begin{array}{lll}
\dot{\eta}(t) & 0 & 0
\end{array}\right\}^{\mathrm{T}}
\end{aligned}
$$

$$
\begin{aligned}
B_{0^{\prime}} \dot{\boldsymbol{\delta}} & =\left\{\begin{array}{lll}
0 & 0 & 0
\end{array}\right\}^{\mathrm{T}} \\
B_{2} \dot{\boldsymbol{\theta}} & =\left\{\begin{array}{lll}
0 & \dot{\theta}(t) & 0
\end{array}\right\}^{\mathrm{T}} \\
B_{4} \dot{\boldsymbol{\alpha}} & =\left\{\begin{array}{lll}
0 & \dot{\alpha}(t) & 0
\end{array}\right\}^{\mathrm{T}} \\
B_{6} \dot{\boldsymbol{\zeta}} & =\left\{\begin{array}{lll}
0 & \dot{\zeta}(t) & 0
\end{array}\right\}^{\mathrm{T}} \\
B_{7} \boldsymbol{\Omega} & =\left\{\begin{array}{lll}
0 & 0 & \Omega(t)
\end{array}\right\}^{\mathrm{T}}
\end{aligned}
$$

Absolute angular velocities when neglecting rotation of celestial objects other than earth:

$$
\begin{array}{ll}
B_{1} \boldsymbol{\omega}_{1}={ }_{B_{1}} \boldsymbol{\Omega}_{\text {earth }}+{ }_{B_{1}} \dot{\boldsymbol{\psi}} & B_{2} \boldsymbol{\omega}_{2}={ }_{B_{2}} \boldsymbol{\omega}_{1}+{ }_{B_{2}} \dot{\boldsymbol{\theta}} \\
{ }_{B_{3}} \boldsymbol{\omega}_{3}={ }_{B_{3}} \boldsymbol{\omega}_{2}+{ }_{B_{3}} \dot{\boldsymbol{\phi}} & { }_{B_{4}} \boldsymbol{\omega}_{4}={ }_{B_{4}} \boldsymbol{\omega}_{3}+{ }_{B_{4}} \dot{\boldsymbol{\alpha}} \\
{ }_{B_{5}} \boldsymbol{\omega}_{5}={ }_{B_{5}} \boldsymbol{\omega}_{4}+{ }_{B_{5}} \dot{\boldsymbol{\beta}} & { }_{B_{6}} \boldsymbol{\omega}_{6}={ }_{B_{6}} \boldsymbol{\omega}_{5}+{ }_{B_{6}} \dot{\boldsymbol{\zeta}} \\
{ }_{B_{7}} \boldsymbol{\omega}_{7}={ }_{B_{7}} \boldsymbol{\omega}_{6}+{ }_{B_{7}} \dot{\boldsymbol{\eta}} & { }_{B_{7}} \boldsymbol{\omega}_{8}={ }_{B_{7}} \boldsymbol{\omega}_{7}+{ }_{B_{7}} \boldsymbol{\Omega}
\end{array}
$$

Velocity and acceleration of rotor relative to housing:

Relative velocity of rotor : $\quad B_{5} \mathbf{v}_{5-7}=\frac{\mathrm{d}}{\mathrm{d} t}\left(B_{5} \mathbf{r}_{\mathbf{5 - 7}}\right)=\{\dot{x}(t) \dot{y}(t) \dot{z}(t)\}^{\mathrm{T}}$

Relative acceleration of rotor : $\quad B_{5} \mathbf{a}_{5-7}=\frac{\mathrm{d}^{2}}{\mathrm{~d} t^{2}}\left({ }_{B_{5}} \mathbf{r}_{5-7}\right)=\{\ddot{x}(t) \ddot{y}(t) \ddot{z}(t)\}^{\mathrm{T}}$ 

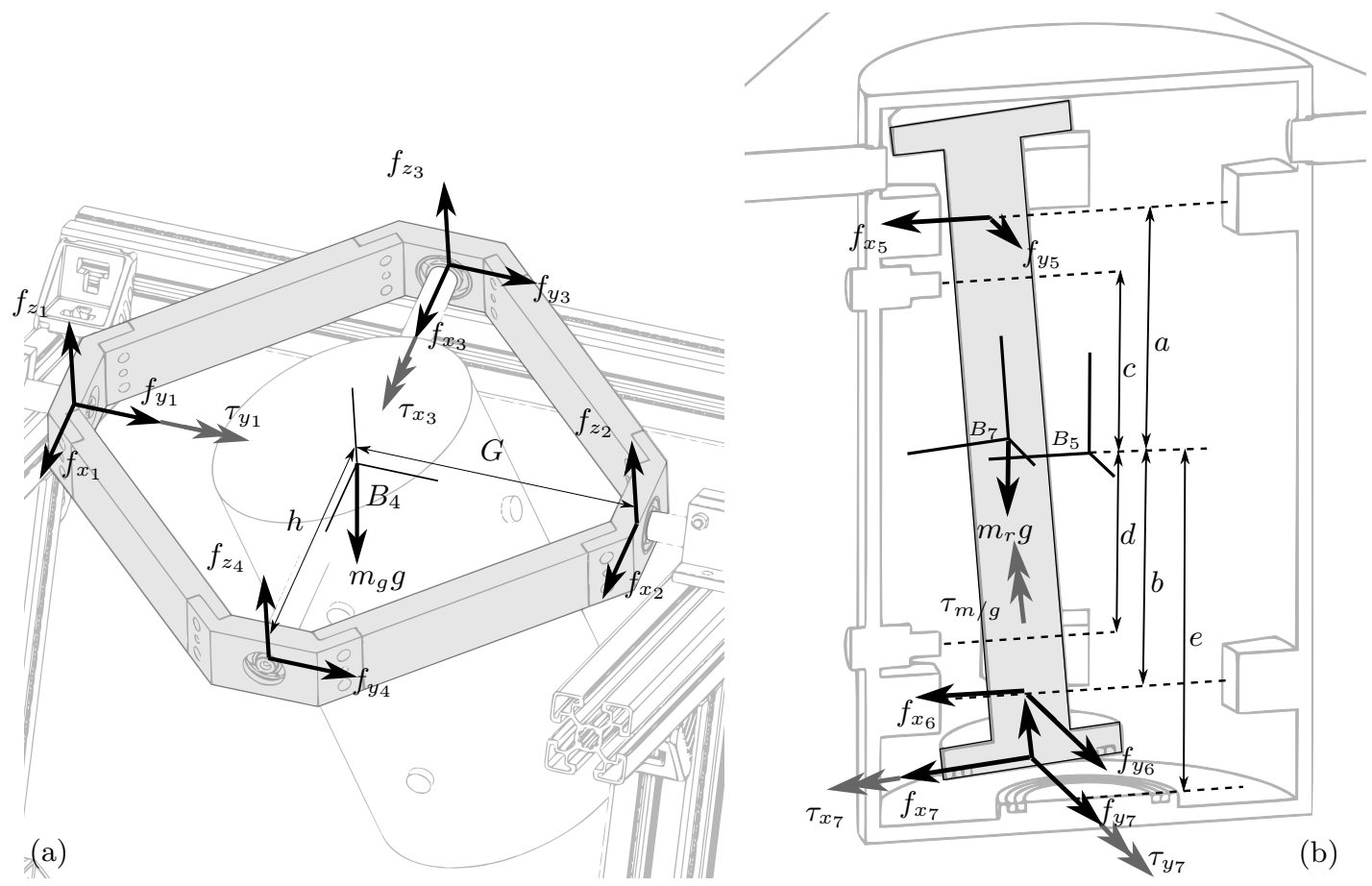

Figure 3: (a): Free-body diagram of gimbal. (b): Free-body diagram of rotor.

Absolute translational accelerations when neglecting accelerations and rotations of all celestial objects including earth:

$$
\begin{aligned}
\text { Vehicle : } & { }_{I} \mathbf{a}_{1}=\left\{\ddot{x}_{1} \ddot{y}_{1} \ddot{z}_{1}\right\}^{\mathrm{T}} \\
\text { Gimbal : } & { }_{I} \mathbf{a}_{4}={ }_{I} \mathbf{a}_{1}+{ }_{I} \dot{\boldsymbol{\omega}}_{3} \times{ }_{I} \mathbf{r}_{3-4}+{ }_{I} \boldsymbol{\omega}_{3} \times\left({ }_{I} \boldsymbol{\omega}_{3} \times{ }_{I} \boldsymbol{r}_{3-4}\right) \\
\text { Housing : } & { }_{I} \mathbf{a}_{5}={ }_{I} \mathbf{a}_{4}+{ }_{I} \dot{\boldsymbol{\omega}}_{5} \times{ }_{I} \mathbf{r}_{4-5}+{ }_{I} \boldsymbol{\omega}_{5} \times\left({ }_{I} \boldsymbol{\omega}_{5} \times{ }_{I} \boldsymbol{r}_{4-5}\right) \\
\text { Rotor : } & { }_{I} \mathbf{a}_{7}={ }_{I} \mathbf{a}_{5}+{ }_{I} \dot{\boldsymbol{\omega}}_{5} \times{ }_{I} \mathbf{r}_{5-7}+{ }_{I} \boldsymbol{\omega}_{5} \times\left({ }_{I} \boldsymbol{\omega}_{5} \times{ }_{I} \boldsymbol{r}_{5-7}\right)+2{ }_{I} \boldsymbol{\omega}_{5} \times{ }_{I} \mathbf{v}_{5-7}+{ }_{I} \mathbf{a}_{5-7}
\end{aligned}
$$

\section{(d) Equations of Motion}

Free-body diagrams of the three bodies can be seen in Figs. 3 and 4 . All bodies are assumed rigid. The 


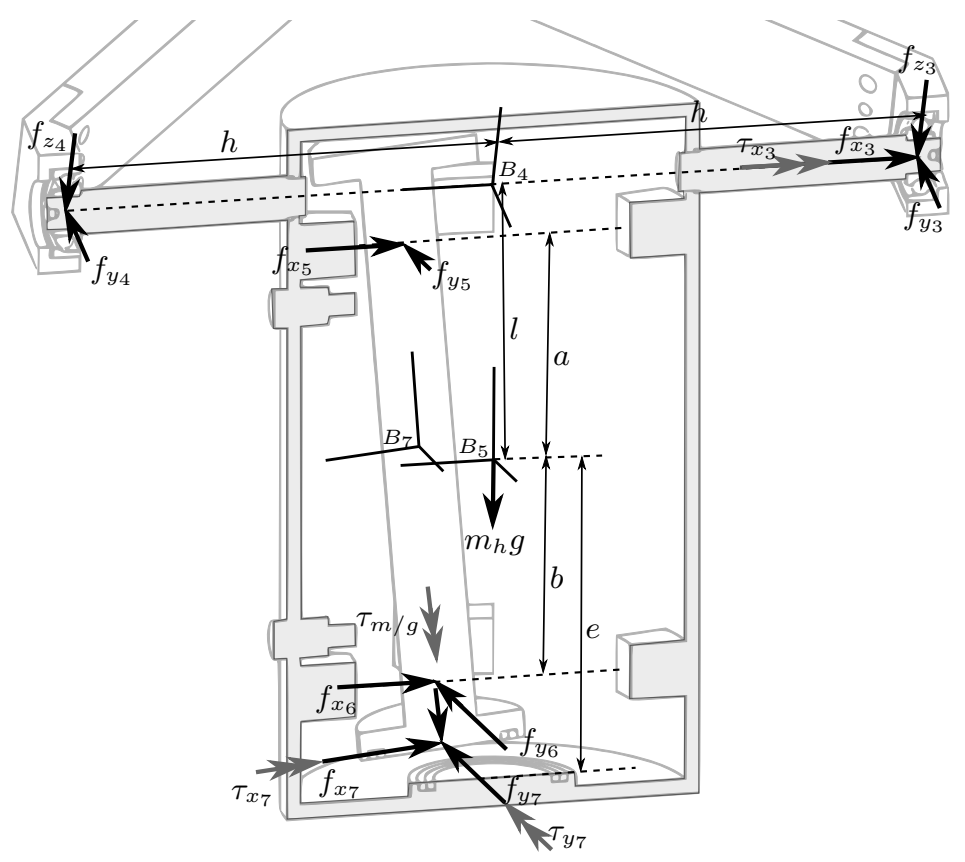

Figure 4: Free-body diagram of housing.

forces and torques in vector form are:

$$
\begin{aligned}
& { }_{B_{4}} \mathbf{f}_{1}=\left\{f_{x_{1}} f_{y_{1}} f_{z_{1}}\right\}^{\mathrm{T}} \\
& { }_{B_{4}} \mathbf{f}_{2}=\left\{\begin{array}{lll}
f_{x_{2}} & 0 & f_{z_{2}}
\end{array}\right\}^{\mathrm{T}} \\
& { }_{B_{4}} \mathbf{f}_{3}=\left\{f_{x_{3}} f_{y_{3}} f_{z_{3}}\right\}^{\mathrm{T}} \\
& { }_{B_{4}} \mathbf{f}_{4}=\left\{\begin{array}{lll}
0 & f_{y_{4}} & f_{z_{4}}
\end{array}\right\}^{\mathrm{T}} \\
& { }_{B_{5}} \mathbf{f}_{5}=\left\{\begin{array}{lll}
f_{x_{5}} & f_{y_{5}} & 0
\end{array}\right\}^{\mathrm{T}} \\
& B_{7} \mathbf{f}_{7}=\left\{f_{x_{7}} f_{y_{7}} f_{z_{7}}\right\}^{\mathrm{T}} \\
& B_{0^{\prime}}, \mathbf{w}_{h}=\left\{\begin{array}{lll}
0 & 0 & m_{h} g
\end{array}\right\}^{\mathrm{T}} \\
& { }_{B_{4}} \boldsymbol{\tau}_{1}=\left\{0 \tau_{y_{1}} 0\right\}^{\mathrm{T}} \\
& { }_{B_{7}} \boldsymbol{\tau}_{7}=\left\{\tau_{x_{7}} \tau_{y_{7}} 0\right\}^{\mathrm{T}}
\end{aligned}
$$

where $m_{g}, m_{h}$, and $m_{r}$ are the masses of the gimbal, housing and rotor. Their mass moment inertia tensors are denoted as ${ }_{B_{4}} \mathbf{I}_{g}, B_{5} \mathbf{I}_{h}$, and ${ }_{B_{5}} \mathbf{I}_{r}$. Inertia of gimbal and housing ${ }_{B_{4}} \mathbf{I}_{g}, B_{5} \mathbf{I}_{h}$ will both be evaluated at gimbal CM while rotor inertia tensor will be evaluated at rotor CM. The torque that is accelerating and decelerating the rotor (from e.g. a motor/generator) is denoted $\boldsymbol{\tau}_{m / g}$. The torques in the gimbal joints $\tau_{y_{1}}$ and $\tau_{x_{3}}$ are caused by friction and will in this work be approximated with

$$
\begin{gathered}
\tau_{y_{1}}=-\mu_{y_{1}} \dot{\alpha}(t) \\
\tau_{x_{3}}=\mu_{x_{3}} \dot{\beta}(t)
\end{gathered}
$$


experimental test rig to be described in a later work. Here, the friction torque was caused mainly by the cables connected to the flywheel rather than the radial load in the joints. It should be noted that (16) is a rough approximation. In a later work, the friction model will be dealt with in more detail. The rotor unbalance $u$ gives rise to the unbalance force

$$
{ }_{B_{7}} \mathbf{f}_{u}=u \Omega^{2}\{\cos (\Omega t) \sin (\Omega t) 0\}^{\mathrm{T}} .
$$

Equations of motion. After describing all reaction forces and absolute accelerations of the three main bodies (gimbal, housing, and rotor), the Newton-Euler Method [28, 29] [30] 31] is used, i. e. dynamic equilibrium in terms of translation and rotation of the three bodies:

Force equilibrium of gimbal:

$$
{ }_{B_{4}} \mathbf{f}_{1}+{ }_{B_{4}} \mathbf{f}_{2}+{ }_{B_{4}} \mathbf{f}_{3}+{ }_{B_{4}} \mathbf{f}_{4}-{ }_{B_{4}} \mathbf{w}_{g}=m_{g} B_{4} \mathbf{a}_{4} .
$$

Moment equilibrium of gimbal around its center of mass:

$$
\begin{array}{r}
B_{4} \mathbf{l}_{g_{1}} \times{ }_{B_{4}} \mathbf{f}_{1}+{ }_{B_{4}} \mathbf{l}_{g_{2}} \times{ }_{B_{4}} \mathbf{f}_{2}+{ }_{B_{4}} \mathbf{l}_{h_{1}} \times{ }_{B_{4}} \mathbf{f}_{1}+{ }_{B_{4}} \mathbf{l}_{h_{2}} \times{ }_{B_{4}} \mathbf{f}_{2}+{ }_{B_{4}} \boldsymbol{\tau}_{1}+{ }_{B_{4}} \boldsymbol{\tau}_{3} \\
={ }_{B_{4}} \mathbf{I}_{g} B_{4} \dot{\boldsymbol{\omega}}_{4}+{ }_{B_{4}} \boldsymbol{\omega}_{4} \times\left({ }_{B_{4}} \mathbf{I}_{g} B_{4} \boldsymbol{\omega}_{4}\right) .
\end{array}
$$

Housing force equilibrium:

$$
-{ }_{B_{5}} \mathbf{f}_{3}-{ }_{B_{5}} \mathbf{f}_{4}+{ }_{B_{5}} \mathbf{f}_{5}-{ }_{B_{5}} \mathbf{f}_{6}-{ }_{B_{5}} \mathbf{f}_{7}-{ }_{B_{5}} \mathbf{w}_{h}=m_{h} B_{5} \mathbf{a}_{5} .
$$

Housing moment equilibrium around gimbal center of mass:

$$
\begin{array}{r}
-{ }_{B_{5}} \mathbf{l}_{h_{1}} \times{ }_{B_{5}} \mathbf{f}_{3}-{ }_{B_{5}} \mathbf{l}_{h_{2}} \times{ }_{B_{5}} \mathbf{f}_{4}-\left({ }_{B_{5}} \mathbf{r}_{4-5}+{ }_{B_{5}} \mathbf{r}_{5-7}+{ }_{B_{5}} \mathbf{r}_{a}\right) \times{ }_{B_{5}} \mathbf{f}_{5} \\
-\left({ }_{B_{5}} \mathbf{r}_{4-5}+{ }_{B_{5}} \mathbf{r}_{5-7}+{ }_{B_{5}} \mathbf{r}_{b}\right) \times{ }_{B_{5}} \mathbf{f}_{6}-{ }_{B_{7}} \mathbf{r}_{e} \times{ }_{B_{7}} \mathbf{f}_{7}-{ }_{B_{5}} \mathbf{r}_{4-5} \times{ }_{B_{5}} \mathbf{w}_{h}-{ }_{B_{5}} \boldsymbol{\tau}_{3}-{ }_{B_{5}} \boldsymbol{\tau}_{7} \\
={ }_{B_{5}} \mathbf{I}_{h B_{5}} \dot{\boldsymbol{\omega}}_{5}+{ }_{B_{5}} \boldsymbol{\omega}_{5} \times\left({ }_{B_{5}} \mathbf{I}_{h B_{5}} \boldsymbol{\omega}_{5}\right)+m_{h B_{5}} \mathbf{r}_{4-5} \times{ }_{B_{5}} \mathbf{a}_{4} .
\end{array}
$$

Rotor force equilibrium:

$$
{ }_{I} \mathbf{f}_{5}+{ }_{I} \mathbf{f}_{6}+{ }_{I} \mathbf{f}_{7}-{ }_{I} \mathbf{w}_{r}+{ }_{I} \mathbf{f}_{u}=m_{r} \mathbf{a}_{7} .
$$

Rotor moment equilibrium around its center of mass:

$$
\begin{array}{r}
B_{7} \boldsymbol{\tau}_{7}+{ }_{B_{7}} \mathbf{r}_{a} \times{ }_{B_{7}} \mathbf{f}_{5}+{ }_{B_{7}} \mathbf{r}_{b} \times{ }_{B_{7}} \mathbf{f}_{6}+{ }_{B_{7}} \mathbf{r}_{e} \times{ }_{B_{7}} \mathbf{f}_{7}+{ }_{B_{7}} \mathbf{r}_{u} \times{ }_{B_{7}} \mathbf{f}_{u} \\
={ }_{B_{7}} \mathbf{I}_{r B_{7}} \dot{\boldsymbol{\omega}}_{8}+{ }_{B_{7}} \boldsymbol{\omega}_{7} \times\left({ }_{B_{7}} \mathbf{I}_{r B_{7}} \boldsymbol{\omega}_{8}\right)
\end{array}
$$

where we assume the rotor to be axisymmetric in order to be able to evaluate the equilibrium in reference frame $B_{7}$.

The multi-body model has been validated against a more simple three degrees if freedom rotating pendulum model and the analytically obtained period of a non-rotating pendulum. In both cases with perfect agreement. This has not been included in the article due to its length and because an experimental validation is to be presented in a later work. 


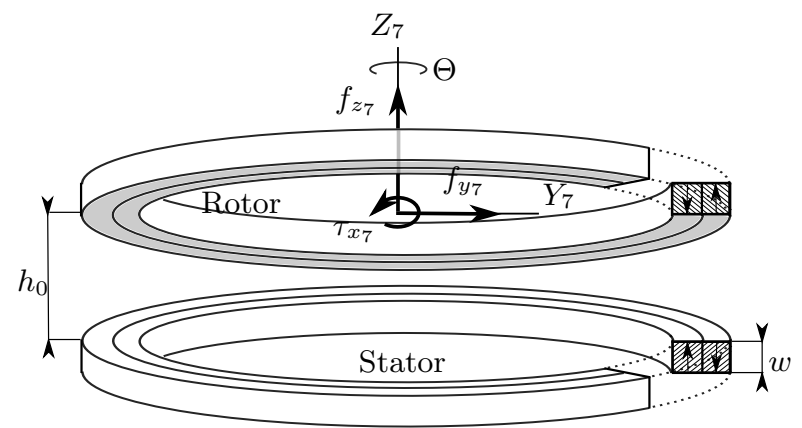

Figure 5: Permanent magnetic bearing (PMB). Both stator and rotor part consist of permanent ring magnets. The magnetic moment of each magnet is illustrated as arrows inside the cross-section of the magnets.

\section{Forces from Permanent Magnet Bearing}

Permanent magnet rings of different diameters are placed coaxially on both rotor and stator as seen in Fig. 5. The magnetic moment of each magnet is illustrated as arrows inside the cross-section of the magnets. If the rotor and stator are perfectly aligned axially, the rotor is lifted vertically and no radial forces are present hence the rotor will be in equilibrium. This state is unstable, however, since the smallest radial displacement $\Delta$ will result in a radial magnetic force pushing the rotor away from the stator center.

The axial force $f_{z_{7}}$ and radial forces $f_{x_{7}}, f_{y_{7}}$ are calculated in two steps. First, the magnetic field density caused by the magnets on the stator is found using the Biot-Savart law. The rotor magnets are in this step completely ignored. Second, the force resulting from the interaction of the rotor magnets and the above magnetic field is calculated using the Lorentz force law. In this step, the stator magnets are ignored except for their resulting magnetic field. To be able to use the above two laws we will represent the magnetization as electric currents $i$ flowing on the surface of the magnet rings. These surface currents are illustrated in Fig. 6 as $i_{1}$ and $i_{2}$. Each surface current is made up of $n_{z}$ small conductors. The magnetic moments of the magnets are:

$$
\begin{array}{ll}
\mathbf{B}_{5} \mathbf{M}_{\mathbf{h}}=\left\{\begin{array}{ll}
00 & 0
\end{array}\right\}^{T} & \text { for the stator } \\
\mathbf{B}_{\mathbf{7}} \mathbf{M}_{\mathbf{r}}=\left\{\begin{array}{lll}
0 & 0 M
\end{array}\right\}^{T} & \text { for the rotor }
\end{array}
$$

The $z$-component of the magnetic moment determines the strength of the current:

$$
i=M \frac{w}{n_{z}}
$$

Where $w$ is height of the magnet. Each current conductor contributes to the magnetic field density $\mathbf{B}_{5} \mathbf{B}$ in its surrounding space. We find the contribution from each current conductor ${ }_{\mathbf{B}_{5}} \mathbf{B}^{\prime}$ in a certain point $R$ using the Biot-Savart law 43

$$
\mathbf{B}_{5} \mathbf{B}^{\prime}=\frac{\mu_{0}}{4 \pi} i \int \frac{B_{5} \mathrm{~d} \mathbf{l}^{\prime} \times B_{5} \hat{\boldsymbol{n}}}{r^{2}}
$$




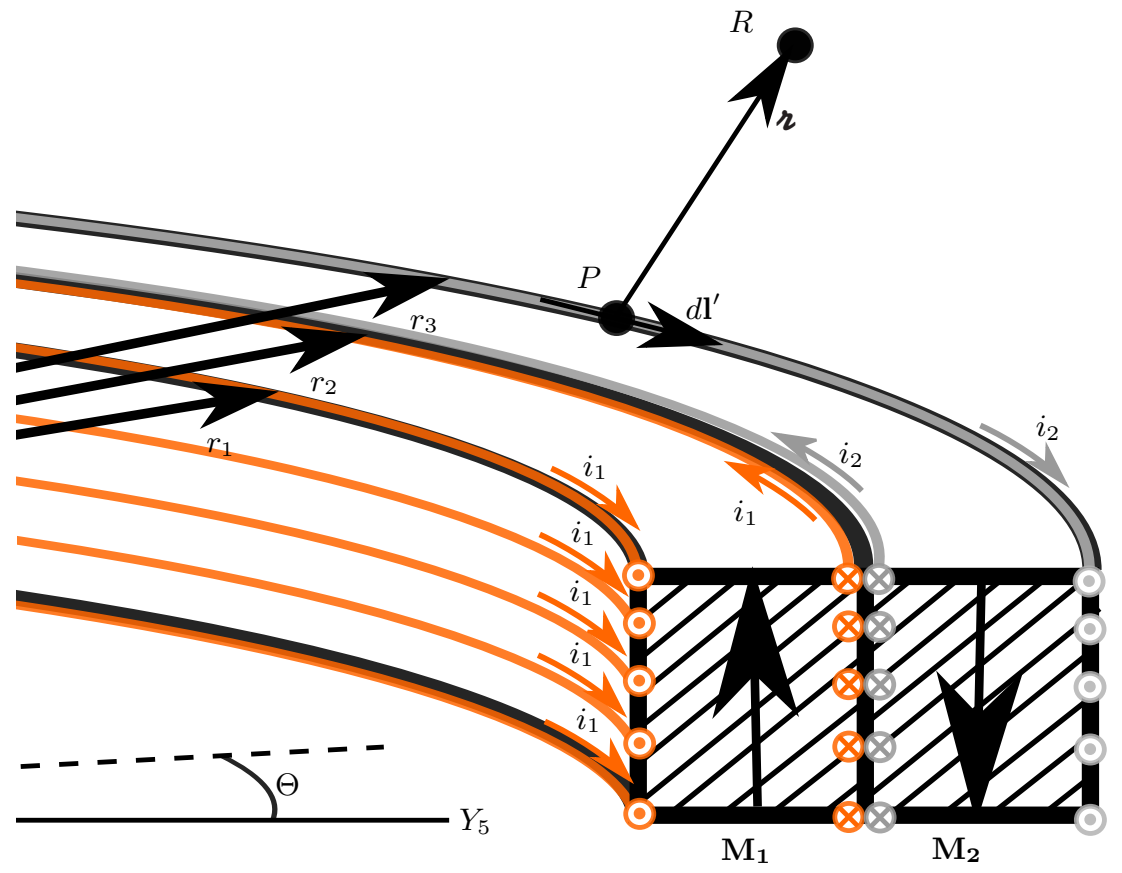

Figure 6: Close-up of ring magnets on the stator part of the PMB. The magnetic moments $\mathbf{M}$ are represented as small current conductors on the surface of the magnets. The radius of the current conductors are denoted $r_{1}, r_{2}$ and $r_{3}$.

where each current conductor is split up into infinitesimal vector elements $\mathrm{dl}^{\prime}$. The permeability of free space is denoted $\mu_{0}$. $\boldsymbol{\imath}$ is the vector from point $P$ to $R$ with direction $\hat{\imath}$ (unit vector) and length $\boldsymbol{r}$. The integral is solved analytically as in [44].

To find the total magnetic field density in a point, we simply add up the contribution from each current conductor.

$$
\mathbf{B}_{5} \mathbf{B}=\sum^{n_{r}} \sum^{n_{z}} \mathbf{B}_{5} \mathbf{B}^{\prime}
$$

where $n_{r}$ is the number of current conductors in a radial direction.

Now when we have the magnetic field density from the stator, we look into how this field affects the rotor magnets. The rotor magnets are represented exactly the same way as the stator - as small current conductors on the surface of the magnets. The force acting on each conductor is found using the Lorentz force law:

$$
\mathbf{B}_{7} \mathbf{f}^{\prime}=i \int\left({ }_{B_{7}} \mathrm{~d} \mathbf{l}^{\prime} \times{ }_{\mathbf{B}_{7}} \mathbf{B}\right)
$$

Note that we do not calculate the magnetic field coming from the rotor magnets - to find the force on the rotor we only use the magnetic field from the stator and we only use the current conductors on the rotor. The total force on the rotor is

$$
{ }_{\mathbf{B}_{7}} \mathbf{f}_{7}=\sum^{n_{r}} \sum^{n_{z}}{ }_{\mathbf{B}_{7}} \mathbf{f}^{\prime} .
$$




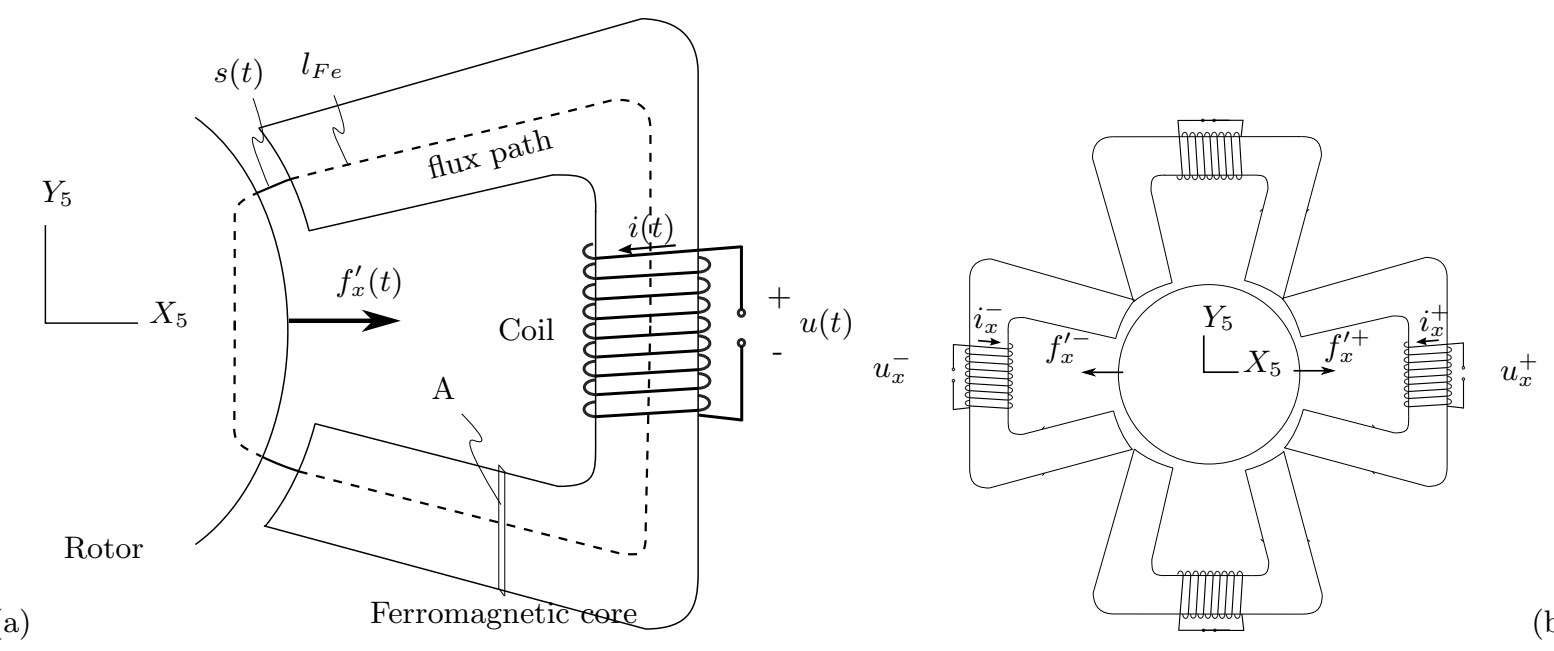

Figure 7: (a): $A M B$ dimensions. (b): Forces in differential setup.

Correspondingly, the torque is:

$$
\begin{gathered}
\boldsymbol{B}_{\mathbf{7}} \boldsymbol{\tau}^{\prime}=i \int\{r \cos (\Theta) r \sin (\Theta) z\}^{\mathrm{T}} \times\left(B_{7} \mathrm{~d} \mathbf{l}^{\prime} \times{ }_{\mathbf{B}_{7}} \mathbf{B}\right) \\
{ }_{B_{7}} \boldsymbol{\tau}_{7}=\sum^{n_{r}} \sum^{n_{z}}{ }_{B_{7}} \boldsymbol{\tau}^{\prime}
\end{gathered}
$$

where $r$ is radius of the current conductor ring.

To be able to implement $\mathbf{f}_{7}$ and $\boldsymbol{\tau}_{7}$ in the multi-body model, Eq. 290 and (31) will be evaluated for many different rotor positions. The results can then be incorporated in the multi-body model either as an interpolated analytical function of rotor position $\mathbf{f}_{7 \text {,interp }}(x, y, z, \eta, \zeta)$ or as a lookup table.

\section{Electromagnetic force and current}

The active magnetic bearings are conventional eight-pole heteropolar bearings. Each bearing consists of four electromagnetic actuators. A sketch of one of the electromagnets can be seen in Fig. 7 a. A coil with current $i$ is wound $n$ times about a ferromagnetic core with relative permeability $\mu_{F e}$ and cross-sectional area $A$. It is assumed that the magnetic flux runs entirely within this area (no leakage) and in the air gaps (no fringing). We combine Ampère's circuital law, Ohm's law, and Faraday's law to obtain a first order differential equation governing the dynamics of the current:

$$
u=R i+\frac{\mu_{0} n^{2} A}{2 s} \frac{\partial i}{\partial t}-\frac{\mu_{0} n^{2} i A}{s^{2}} \frac{\partial s}{\partial t}
$$

where $R$ is resistance. Furthermore, using the principle of virtual work, the magnetic force can be expressed as:

$$
f_{x}^{\prime}=\mu_{0} A\left(\frac{n i}{2 s}\right)^{2} \cos \left(\frac{\pi}{8}\right)
$$


where the factor $\cos \left(\frac{\pi}{8}\right)$ is present because each leg on the electromagnet is slightly angled relative to the $X_{5}$-axis. For an eight-pole heteropolar magnetic bearing, the angle is $\frac{\pi}{8}$. Equations $(32)$ and $(33)$ are based on the assumptions that the rate of change of electric flux $\frac{\mathrm{d} \Phi_{E}}{\mathrm{~d} t}$ (change of current with time) contributes insignificantly to the magnetic field (magnetostatic approximation) thus eddy-current effects are neglected. Also, it has been assumed that $\mu_{F e} \gg \mu_{0}$ and a constant flux density over the cross-section area; $\Phi=B A$. Finally the effect of magnetic saturation has been neglected. A derivation of eqs. $(32)$ and $(33)$ is found in [45].

We express the air gap as $s=x_{0}-x_{b}$. The rotor radial displacements at the bearings $x_{b}$ and $y_{b}$ are described for bearing $\mathrm{A}$ and $\mathrm{B}$ using basic trigonometry as:

$$
\begin{array}{ll}
x_{b_{A}}=x+\tan \zeta(a-z) & y_{b_{A}}=y-\frac{\tan \eta}{\cos \zeta}(a-z) \\
x_{b_{B}}=x-\tan \zeta(b+z) & y_{b_{B}}=y+\frac{\tan \eta}{\cos \zeta}(b+z)
\end{array}
$$

The total $X_{7}$-directional force from the actuator consists of the forces from two electromagnets as seen in Fig. 7b. The final expressions for the electromagnetic forces become:

$$
\begin{gathered}
f_{x_{5}}=\frac{\mu_{0}}{4} A \cos \left(\frac{\pi}{8}\right)\left[-\left(\frac{n i_{x_{A}}^{-}}{\left(x_{0}+x_{b_{A}}\right)}\right)^{2}+\left(\frac{n i_{x_{A}}^{+}}{\left(x_{0}-x_{b_{A}}\right)}\right)^{2}\right] \\
f_{y_{5}}=\frac{\mu_{0}}{4} A \cos \left(\frac{\pi}{8}\right)\left[-\left(\frac{n i_{y_{A}}^{-}}{\left(y_{0}+y_{b_{A}}\right)}\right)^{2}+\left(\frac{n i_{y_{A}}^{+}}{\left(y_{0}-y_{b_{A}}\right)}\right)^{2}\right]
\end{gathered}
$$

$$
\begin{aligned}
& f_{x_{6}}=\frac{\mu_{0}}{4} A \cos \left(\frac{\pi}{8}\right)\left[-\left(\frac{n i_{x_{B}}^{-}}{\left(x_{0}+x_{b_{B}}\right)}\right)^{2}+\left(\frac{n i_{x_{B}}^{+}}{\left(x_{0}-x_{b_{B}}\right)}\right)^{2}\right] \\
& f_{y_{6}}=\frac{\mu_{0}}{4} A \cos \left(\frac{\pi}{8}\right)\left[-\left(\frac{n i_{y_{B}}^{-}}{\left(y_{0}+y_{b_{B}}\right)}\right)^{2}+\left(\frac{n i_{y_{B}}^{+}}{\left(y_{0}-y_{b_{B}}\right)}\right)^{2}\right] .
\end{aligned}
$$

The AMB forces have been compared with results obtained using the finite element method. This has been done in the program FEMM [46]. The results were in good agreement which confirms the validity of the assumptions made for the above derivation.

\section{Controller}

Each magnetic actuator will be controlled based on the rotor position and current in the specific actuator and it will be completely independent on positions and currents in the other actuators (decentralised). The decentralised controller configuration applied on each actuator can be seen in Fig. 8 A proportional-integralderivative controller $C_{P I D}$ uses the measured rotor position $x_{\mathrm{se}}$ to determine reference currents $i_{\text {ref }}^{-}$and $i_{\text {ref }}^{+}$. 


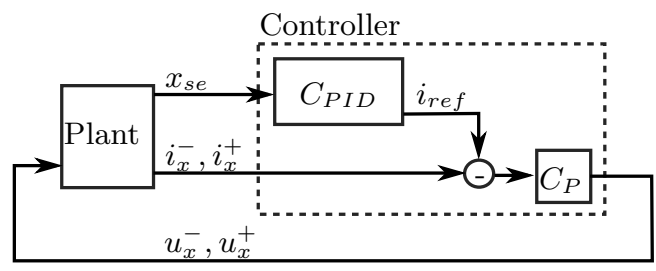

Figure 8: Controller configuration for each actuator (decentralised).

The measured rotor positions are given as:

$$
\begin{array}{ll}
x_{\mathrm{se}_{A}}=x+\tan \zeta(c-z) & y_{\mathrm{se}_{A}}=y-\frac{\tan \eta}{\cos \zeta}(c-z) \\
x_{\mathrm{se}_{B}}=x-\tan \zeta(d+z) & y_{\mathrm{se}_{B}}=y+\frac{\tan \eta}{\cos \zeta}(d+z)
\end{array}
$$

A proportional controller $C_{P}$ outputs voltage $u^{-}$and $u^{+}$based on the difference between the reference current and measured current. The controller $C_{P}$ can be seen as a simple power amplifier.

This way we have confined the controller to be almost as simple as possible while at the same time representing the configuration most widely used. The configuration can be expressed as:

$$
\begin{aligned}
C_{P}: & u_{x}^{ \pm}=K_{c}\left(i_{\text {ref }}^{ \pm}-i_{x}^{ \pm}\right) \\
C_{P I D}: & i_{\text {ref }}^{ \pm}=i_{\text {bias }} \mp K_{p}\left(T_{d} \dot{x}_{\text {se }}+x_{\text {se }}+K_{i} \int x_{\mathrm{se}} \mathrm{d} t\right)
\end{aligned}
$$

where $i_{\text {bias }}$ is a constant current applied to both electromagnets and $K_{c}, K_{p}, T_{d}$, and $K_{i}$ are controller constants. Combining equations $(38),(39)$, and $(32)$ we obtain the final equation for the coil dynamics:

$$
\begin{array}{r}
R i_{x}^{ \pm}+\frac{\mu_{0} n^{2} A}{2\left(x_{0} \mp \cos (\pi / 8) x_{b}\right)} \dot{i}_{x}^{ \pm} \pm \frac{\mu_{0} n^{2} i_{x}^{ \pm} A}{\left(x_{0} \mp \cos (\pi / 8) x_{b}\right)^{2}} \cos \left(\frac{\pi}{8}\right) \dot{x}_{b} \\
=K_{c}\left(i_{\text {bias }} \mp K_{p}\left(T_{d} \dot{x}_{\mathrm{se}}+x_{\mathrm{se}}+K_{i} e_{x_{\mathrm{se}}}\right)-i_{x}^{ \pm}\right)
\end{array}
$$

where $\int x_{\mathrm{se}} \mathrm{d} t$ is replaced with $e_{x_{\mathrm{se}}}$ such that

$$
\dot{e}_{x_{\mathrm{se}}}=x_{\mathrm{se}}
$$

which can readily be implemented in a numerical time integration solver.

When implementing the controller in real life, the PID-controller, eq. 390 is usually realised by limiting the bandwidth of the derivative term. Furthermore it is hard to avoid phase and time delays of some significant influence; for example, from signal conditioning and digital sample delays. We will approximate these phase and time delays with a second order low-pass filter applied to the measured rotor position. We denote the unfiltered measurement as $x_{\mathrm{se}}^{\prime}$ and the filter cut-off frequency $w_{c}$ to obtain the relation:

$$
\ddot{x}_{\mathrm{se}}=w_{c}^{2} x_{\mathrm{se}}^{\prime}-w_{c}^{2} x_{\mathrm{se}}-2 \zeta w_{c} \dot{x}_{\mathrm{se}}
$$




\section{Theoretical Results}

This section gives examples on how to utilise the above theoretical model to investigate the significance of various key design parameters. The input parameters used in the following are given in Appendix A. When modelling AMBs, it is common practice to linearise the AMB forces $f_{x_{5}}, f_{y_{5}}, f_{x_{6}}$, and $f_{y_{6}}$, as well as the coil dynamics eq. 40 and (41). For the input parameters at hand we have compared results with and without linearised AMB equations (equations of motion are kept non-linear). The comparison is carried out by simulating the system in time and perturbing the rotor with a step force on $f_{x_{5}}$ and $f_{x_{6}}$, effectively displacing the rotor radially with approximately $100 \mu \mathrm{m}$ from the linearisation point. The force is created by stepping $i_{\text {ref }, x_{A}}$ and $i_{\text {ref }, x_{B}}$ from $0 \mathrm{~A}$ to $0.05 \mathrm{~A}$. The housing is kept stationary. In this case, $100 \mu \mathrm{m}$ is $50 \%$ of the backup bearing clearance, $20 \%$ of the AMB nominal gap, and is chosen because it is larger than any realistic displacement the rotor will experience. It is found that the maximum difference in rotor displacement between the non-linear and linear cases are below $1 \%$. In order to further simplify the model, it is also often common practice to completely neglect the coil dynamics and assume that the current perfectly follows the reference current $i=i_{\text {ref. }}$ Therefore, simulations are also carried out using this simplification. Again, displacing the rotor about $100 \mu \mathrm{m}$, a maximum difference of $9 \%$ is found when the coil dynamics are neglected. This means that if it is assumed that $i=i_{\text {ref }}$, then the model becomes less capable of capturing the true dynamics of the current. Due to the low difference between non-linear and linear AMB equations of less than $1 \%$, the linear AMB equations were chosen. The linearised equations are given in Appendix B. Due to the significant difference when neglecting coil dynamics of $9 \%$ it was decided to keep the coil dynamics in the model.

Another non-linear effect that may or may not be significant is the magnetic saturation of the ferromagnetic core. This has been dealt with in detail in [47. The significance of magnetic saturation has been assessed for the current applications. In the examples to follow, the bias current is kept at $i_{\text {bias }}=0.6 \mathrm{~A}$ and thus the operational span of the total current (the sum of control and bias current) is $0-1.2$ A. Following the method described in [48, it is found that this operational span is well within the linear region of the B-H curve (relation between magnetic intensity and flux). That is, non-linear effects coming from magnetic saturation are avoided. The B-H curves for different frequencies of magnetic intensity $H$ are obtained from 49. The control currents, $i_{x_{A}}, i_{x_{B}}, i_{y_{A}}, i_{y_{B}}$ will be present as state variables in the mathematical model, thus they are readily available for monitoring. If any of them are exceeding the bias current, the simulations will become inaccurate. In all the examples to follow, it is found that they are not exceeding the bias current.

The force from the permanent magnet bearing $\mathbf{f}_{7}$ is investigated for the input parameters at hand. The force as a function of rotor position can be seen in Fig. 9. The results are compared with the results obtained using the commercial software COMSOL, which uses the magnetic vector potential approach and the finiteelement method to find magnetic field density and resulting forces [50, [51] 46. The results show good 


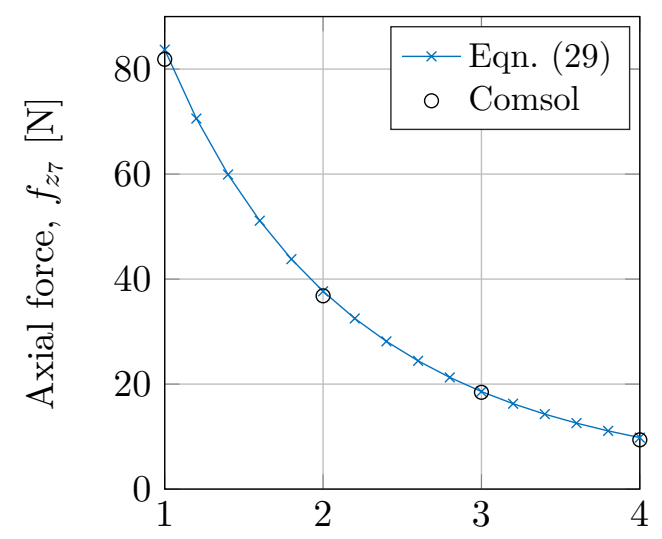

(a)
Axial air gap, $h_{0}[\mathrm{~mm}]$

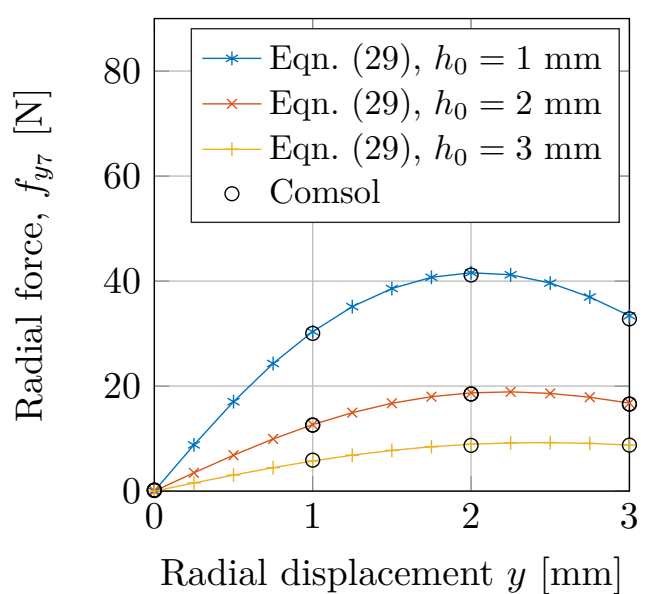

(b)

Figure 9: PMB forces as a function of rotor position. (a): Axial force. (b): Radial force.

agreement as seen in the figure (please see Appendix D for COMSOL solver settings). Considering that the rotor is moving less than $100 \mu \mathrm{m}$ in both axial and radial directions it is assessed that an approximate linear relationship between displacement and permanent magnet force is sufficient to capture the behaviour of the permanent magnet forces.

$$
\begin{aligned}
& f_{x_{7}}=-k_{r}(x-\zeta e) \\
& f_{y_{7}}=-k_{r}(y+\eta e) \\
& f_{z_{7}}=-k_{z}\left(z-z_{0}\right)
\end{aligned}
$$

where $k_{r}=-20 \mathrm{kN} / \mathrm{m}$ and $k_{z}=40 \mathrm{kN} / \mathrm{m}$. In addition to the results from Fig. 9, it is assessed how the torques $\tau_{x_{7}}$ and $\tau_{y_{7}}$ are increasing with rotor tilt $\eta$ and $\zeta$. It is found that the torques are insignificantly small given the interval of tilt angles.

All of the following results are obtained by setting up Eq. $(18)-(23)$, Eq. $($ B.9 $)-(B .12)$, and Eq. (41) in a system of equations

$$
\mathbf{A}(\mathbf{t}) \mathbf{x}(\mathbf{t})=\mathbf{b}(\mathbf{t})
$$

which is solved for

$$
\mathbf{x}(\mathbf{t})=\left\{\ddot{x} \ddot{y} \ddot{z} \ddot{\eta} \ddot{\zeta} \dot{\Omega} \dot{i}_{x_{A}} \dot{i}_{y_{A}} \dot{i}_{x_{B}} \dot{i}_{y_{B}} \dot{e}_{x_{\mathrm{se}_{A}}} \dot{e}_{y_{\mathrm{se}_{A}}} \dot{e}_{x_{\mathrm{se}_{B}}} \dot{e}_{y_{\mathrm{se}_{B}}} f_{x_{1}} f_{y_{1}} f_{z_{1}} f_{x_{2}} f_{z_{2}} f_{x_{3}} f_{y_{3}} f_{z_{3}} f_{y_{4}} f_{z_{4}} \ddot{\alpha} \ddot{\beta}\right\}^{\mathrm{T}} .
$$

The system is integrated in time using an explicit Runge-Kutta scheme. The system matrix $\mathbf{A}(\mathbf{t})$ and the right-hand side $\mathbf{b}(\mathbf{t})$ are given in Appendix C

From solution $\mathbf{x} 47$ it becomes clear how the model provides a powerful tool for analysing vibrations and the stability of the system with crucial dynamics and forces being determined: the rotor translational and rotational movements $\ddot{x} \ddot{y} \ddot{z} \ddot{\eta} \ddot{\zeta} \dot{\Omega}$, the electrodynamics of the magnetic coils $\dot{i}_{x_{A}} \dot{i}_{y_{A}} \dot{i}_{x_{B}} \dot{i}_{y_{B}}$, as well as the 
gimbal dynamics $\ddot{\alpha} \ddot{\beta}$, and the reciprocating forces in the gimbal mount $f_{x_{1}} f_{y_{1}} f_{z_{1}} f_{x_{2}} f_{z_{2}} f_{x_{3}} f_{y_{3}} f_{z_{3}} f_{y_{4}} f_{z_{4}}$. The input design parameters which make up $\mathbf{A}(\mathbf{t})$ and $\mathbf{b}(\mathbf{t})$ are summarised (see Sec. 2 for full description):

$$
\begin{array}{r}
\Omega_{\text {earth }}, \delta, \psi(t), \theta(t), \phi(t), x_{3-4}, y_{3-4}, z_{3-4}, l, a, b, c, d, e, G, h, \mu_{y_{1}}, \mu_{x_{3}}, u, l_{u}, m_{r}, \\
m_{h}, m_{g}, I_{r_{p}}, I_{h_{p}}, I_{g_{p}}, I_{r_{t}}, I_{h_{t}}, I_{g_{t}}, \tau_{m / g} M, w, n_{z}, n_{r}, r, A, n, i_{\text {bias }}, x_{0}, K_{c}, K_{p}, T_{d}, K_{i}
\end{array}
$$

where $I_{r_{p}}, I_{h_{p}}, I_{g_{p}}, I_{r_{t}}, I_{h_{t}}, I_{g_{t}}$ are polar and transversal mass moments of inertias for rotor, housing, and gimbal respectively.

As this work is focusing on how to obtain the model and not how to apply it to a specific case, we will not do a full parametric study on the relation of all the input parameters to all outputs. Such a parametric study would require the description of a specific case with FESS design requirements and a specification of critical vehicle movements. This deserves its own work especially considering the number of inputs and outputs. Furthermore, choosing the optimal control parameters $K_{c}, K_{p}, T_{d}, K_{i}$ or a whole other type of controller is a study of its own; this article will not focus on the control parameters, although the model will indeed be useful for model based control design.

Instead, this article will limit itself to a few selected results aimed at motivating the model and exemplifying how the different physical design parameters affect dynamics. In the following, the effects of the rotation of earth are neglected although it was demonstrated how to include it in Sec. 2

\subsection{Example 1 - Comparison With and Without Gimbal}

In the first example we latch the gimbal such that $\alpha$ and $\beta$ are forced to be zero and the flywheel is moving with the vehicle. The vehicle is rolling with an amplitude of $45^{\circ}$ and a period of $12 \mathrm{~s}$. This motion is taken from 52] in which a naval FESS is required to withstand this motion. We are interested in the maximum force reciprocated through the AMBs which is the gyroscopic force occurring in the $X_{5}$-direction (perpendicular to the axis of the roll). In Fig. 10, this force is shown for the top bearing for three different rotor polar mass moments of inertia $I_{r_{p}}$. The nominal $I_{r_{p}}$ is $0.0038 \mathrm{~m}^{2} \mathrm{~kg}$. We only look at the steady-state response. As seen, the force increases with increasing $I_{r_{p}}$. This is as expected from the last term on the left-hand side of eq. 23). Essentially, this example just serves to demonstrate the gyroscopic force coming from the last term on the left-hand side of eq. 23). This might seem trivial, however as seen in Fig. 10 this gyroscopic force is, as $I_{p_{r}}$ is increasing, rapidly becoming large which is the reason for introducing the gimbal mount. In this case, $I_{p_{r}}$ and $\Omega$ are conservatively small compared to a realistic flywheel system of the same size. The main objective of designing the FESS is to maximise kinetic energy stored and thus increase $I_{r_{p}}$ and $\Omega$. Therefore it is likely that the gyroscopic force is much larger in a real system of the same size.

Fig. 11 compares AMB forces with and without gimbal mount. Again, we only look at the steady-state response - although this was difficult to obtain from the gimballed case as seen in Fig. 11b. When the gimbal mount is present the lateral motions of the flywheel are extremely small resulting in the AMB forces also 


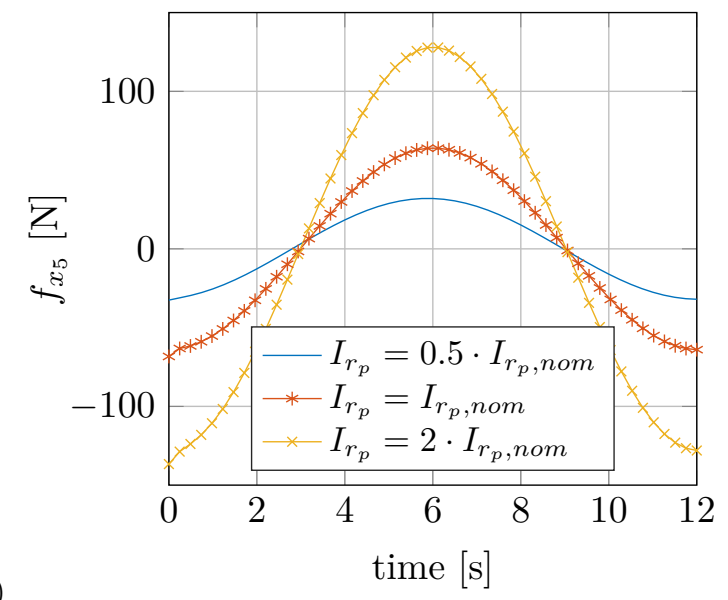

Figure 10: Forces in active magnetic bearings when vehicle is rolling and no gimbal is present such that flywheel is also rolling. The nominal $I_{r_{p}}$ is $0.0038 \mathrm{~m}^{2} \mathrm{~kg}$.

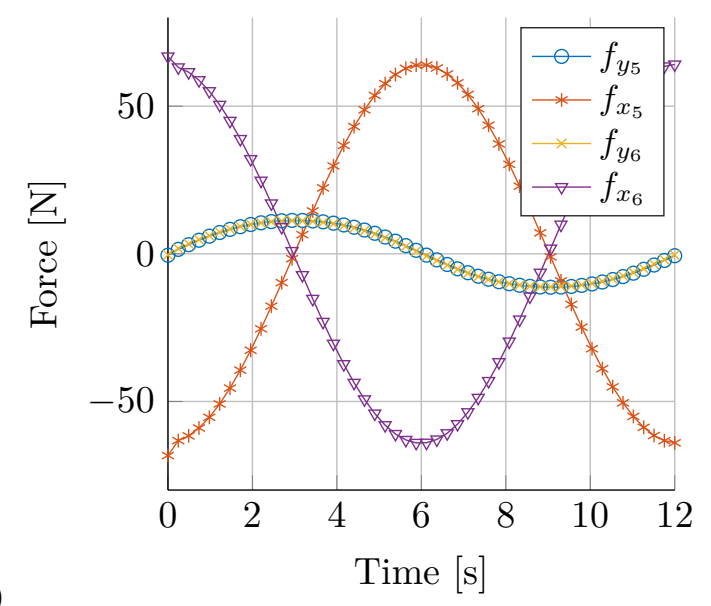

(a)

(a)

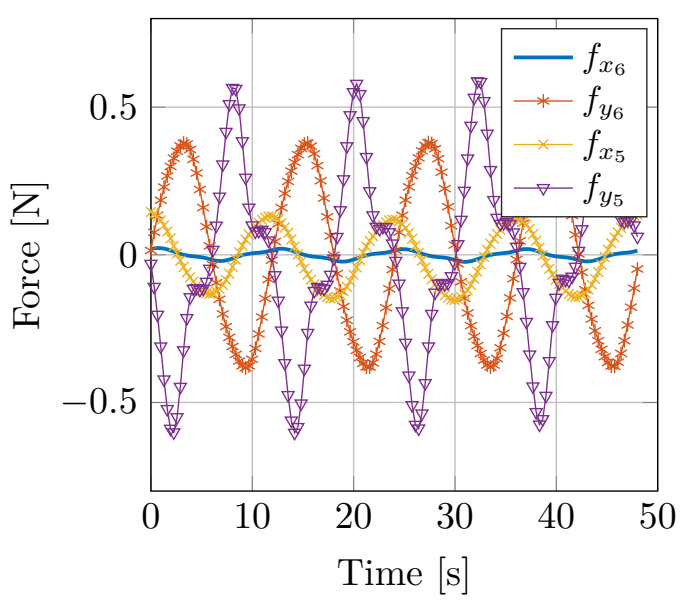

Figure 11: AMB forces when vehicle is rolling. $I_{r_{p}}=0.0038 \mathrm{~m}^{2} \mathrm{~kg}$. (a): Without Gimbal. (b): With Gimbal.

being very small. The only reason the flywheel is still moving slightly is the friction in the gimbal bearings and the fact that the flywheel is not placed directly on the roll axis of the vehicle.

\subsection{Example 2 - Unbalance Response}

In this example we want to investigate how the rotor moves relative to the housing due to unbalance. The unbalance amplitude $u$ is $6.4 \mathrm{~g} \cdot \mathrm{mm}$ which corresponds to the G 6.3 grade of the ISO 1940 standard 53 for a rotor of the given mass with a maximum rotational speed of $30000 \mathrm{RPM}$. The location of the unbalance is set at the extremity of the rotor $l_{u}=0.15 \mathrm{~m}$. Time simulations are carried out for different rotational speeds and different ratios of transversal mass moments of inertia of housing and rotor $I_{h_{t}} / I_{r_{t}}$ and of gimbal and rotor $I_{g_{t}} / I_{r_{t}}$. The different ratios are obtained by keeping $I_{r_{t}}$ fixed and changing $I_{h_{t}}$ and $I_{g_{t}}$. For each simulation, 
the maximum steady-state displacement amplitude at the location of the sensors $x_{\mathrm{se}_{A}}, x_{\mathrm{se}_{B}}, y_{\mathrm{se}_{A}}, y_{\mathrm{se}_{B}}$ are noted as well as the maximum steady-state amplitude of the AMB forces $f_{x_{A}}, f_{y_{A}}, f_{x_{B}}, f_{y_{B}}$. The maximum amplitude for all the different simulations is then plotted in Fig. 12 for displacement and Fig. 13 for forces. Both figures show a peak in amplitude at the resonance frequency at around 400-500 rounds per minute (RPM). The amplitude of the peak is 6-11 $\mu \mathrm{m}$ and is strongly dependant on the ratios $I_{h_{t}} / I_{r_{t}}$ and $I_{g_{t}} / I_{r_{t}}$.

For a low $I_{h_{t}}$ and/or $I_{g_{t}}$ and thus small ratios, the response is small. The peak is largest when the ratios reach $I_{h_{t}} / I_{r_{t}}=1, I_{g_{t}} / I_{r_{t}}=0.1$. Note that this case does not give exactly the same displacement as the case $I_{h_{t}} / I_{r_{t}}=0.1, I_{g_{t}} / I_{r_{t}}=1$ although the sum of the two ratios $I_{h_{t}} / I_{r_{t}}$ and $I_{g_{t}} / I_{r_{t}}$ is the same. The force amplitudes are, interestingly enough, even more distinct in the two cases as seen in Fig. 13 .

From both figures it can be seen that the location of the peak and thus the resonance frequency is also changing slightly with the ratios.

The two figures 12 and 13 look much alike due to the fact that the forces are controlled based on feedback from the radial rotor position. The forces are however, unlike the displacements, increasing at higher rotational velocity because the forces are also controlled based on feedback from the radial rotor velocity which is increasing with increasing rotational velocity. In a real case however, this high control effort at high rotational velocity is usually removed by an unbalance control algorithm [54. Finally, when looking at the forces in Fig. 13 one can conclude that their magnitudes are small and thus the force requirements due to unbalance are, in this case small.

In summary, the results show how the dynamics of the system are significantly changing with the dimensions of the bodies i.e. gimbal, housing, and rotor. Thus the model can be used to ensure that undesirably large rotor displacements or forces due to unbalance are avoided.

\subsection{Example 3 - External Perturbation}

This example deals with how the rotor and housing moves when the vehicle is accelerating translationally forward (surging). The surge acceleration is on $0.25 \mathrm{~g}$ which is taken from 52 in which a naval FESS is required to withstand this motion. Time simulations are carried out for different lengths $l$ between the gimbal pivot point and the rotor-housing center of mass. The maximum transient amplitude of the relative movement of rotor and housing as well as the maximum transient amplitude of the AMB forces are noted. Furthermore, the maximum transient amplitude of the rotation of the housing $\alpha$ and the maximum transient force in the gimbal joints are noted.

In Fig. 14a, we see how the maximum displacement of rotor relative to housing is dependent on the length between pivot point and CM, $l$. The largest displacement occurs when $l$ is around $50 \mathrm{~mm}(16 \%$ of rotor length). When looking at the effect of the ratio $I_{h_{t}} / I_{r_{t}}$ it is seen that for small $I_{h_{t}} / I_{r_{t}}$ the largest displacement of $30 \mu \mathrm{m}$ occurs. This is well within the maximum allowable displacement which is given as the clearance of the backup bearing, usually between $100-200 \mu \mathrm{m}$. The AMB forces (Fig. 14b) show exactly 


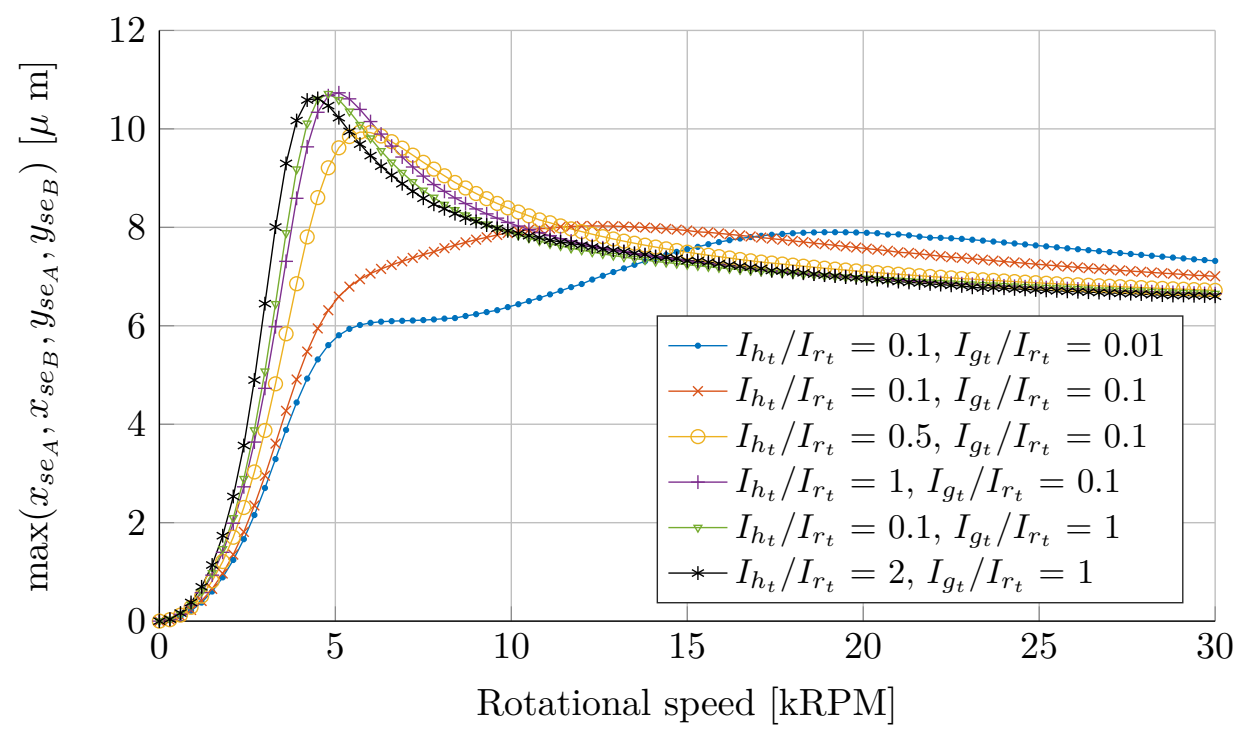

Figure 12: Maximum rotor displacement due to unbalance response in a gimballed flywheel system.

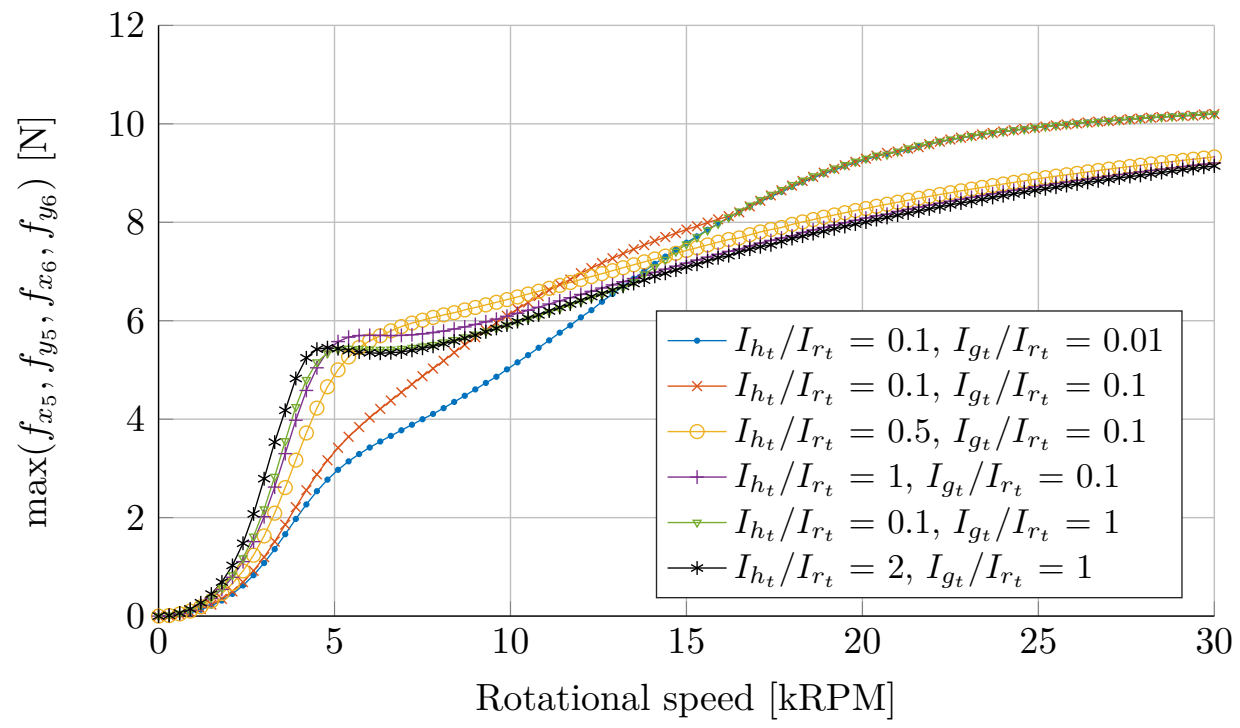

Figure 13: Maximum AMB force due to unbalance response in a gimballed flywheel system. 


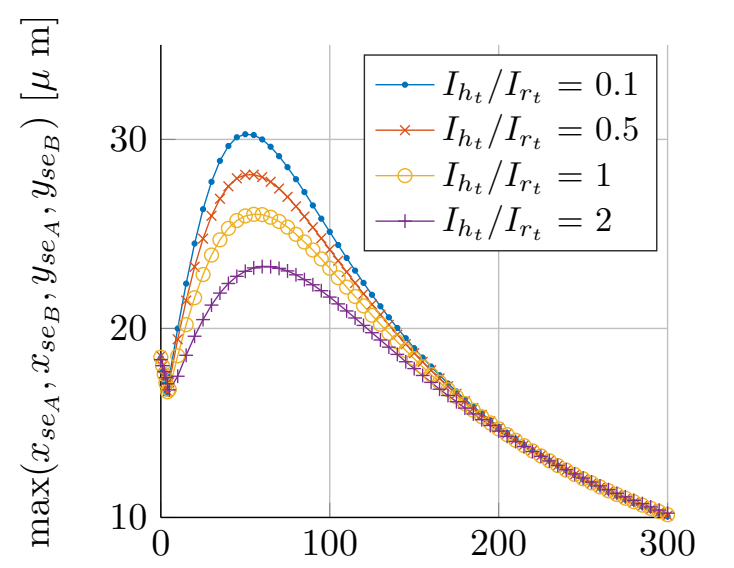

(a)

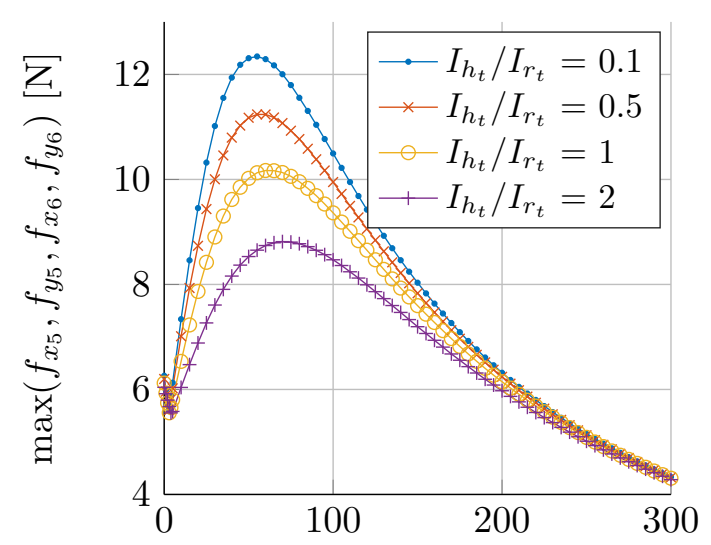

Length from pivot point to CM, $l[\mathrm{~mm}]$

Figure 14: Response of rotor relative to housing due to outer acceleration in a gimballed flywheel system. (a): Maximum rotor displacement. (b): Maximum AMB forces.

the same behaviour as the displacement. From the forces we also see that they are small compared to the

\section{Conclusion and Future Aspects}

A mathematical model has been derived which can be used to investigate the interacting motions and forces of a FESS comprising of rotor, magnetic bearings, housing and a passive gimbal mount. The model uses as inputs: the physical design parameters of the three bodies, the active magnetic bearings, and the 


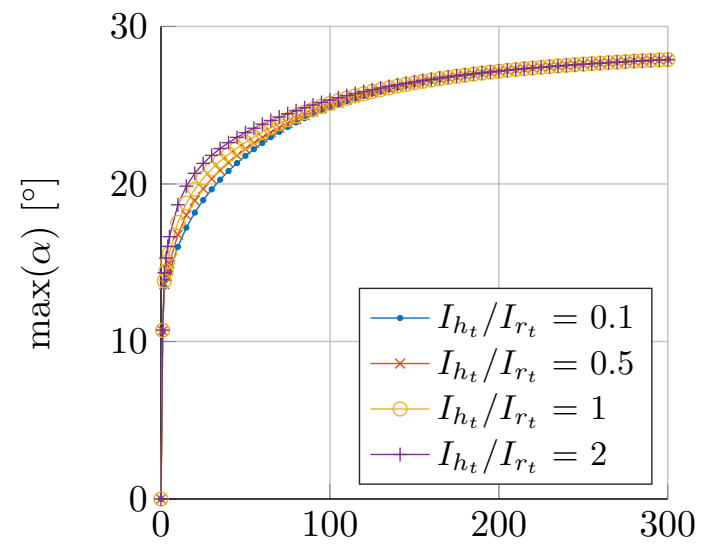

(a)

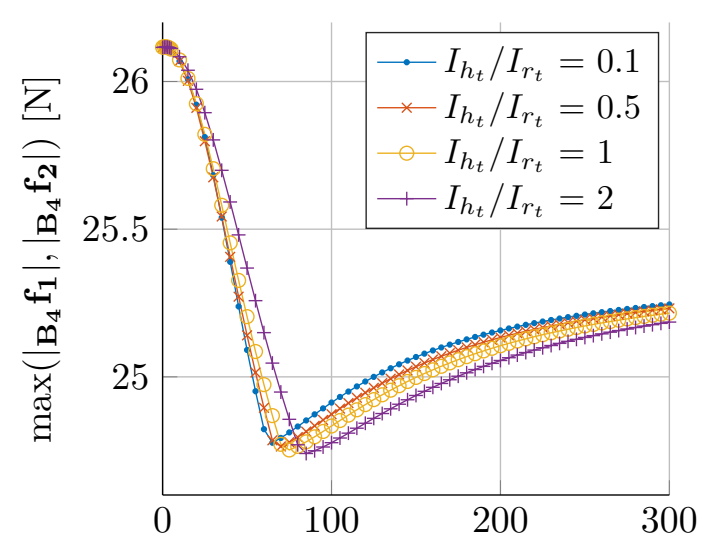

Length from pivot point to CM, $l[\mathrm{~mm}]$

Figure 15: Response in gimbal joints due to outer acceleration in a gimballed flywheel system. (a): Maximum swing of housing. (b): Maximum gimbal joint force.

permanent magnet bearings, as well as the control parameters of the active magnetic bearings, and finally

the motions of the vehicle. The model gives as outputs: the movements of the three bodies, the active magnetic bearing coil currents, and the forces reciprocated through the system.

The model is used to simulate a specific flywheel energy storage system. For the system parameters used, linearised AMB equations deviate from the non-linear equations with less than $1 \%$ motivating the use of the linearised equations. Neglecting coil dynamics gives an error of $9 \%$ which motivates not neglecting coil dynamics. The forces in the permanent magnet bearing show good agreement with results from commercial software COMSOL. The forces behave approximately linearly within the relevant interval of rotor displacement.

In the investigated flywheel system, the forces in the non-gimballed flywheel in the nominal case reach 64 N. In comparison, by implementing a gimbal mount, the forces are reduced to $19 \%$ of the non-gimballed case. The largest forces occurring in a gimballed system are due to unbalance and vehicle acceleration. These forces reach approximately $10 \mathrm{~N}$ and $12 \mathrm{~N}$ respectively. Thus it is likely that if the flywheel is not gimbalmounted, the gyroscopic force will be significantly larger than the other forces in the magnetic bearings; especially considering that the gyroscopic force will increase with increasing polar mass moment of inertia and rotational velocity and that these two parameters are strongly incited to be as large as possible in order to increase kinetic energy. When comparing the non-gimballed flywheel with the gimballed equivalent it is seen how the gimbal mount effectively removes large gyroscopic forces.

An unbalance response of G 6.3 is investigated for different ratios of transversal mass moment of inertia of housing, gimbal, and rotor. The maximum displacement is around $11 \mu \mathrm{m}$ which is only $6 \%$ of the maximum allowable displacement of $200 \mu \mathrm{m}$. It is found that the response is strongly dependent on the ratios of mass 
moments of inertia. The forces in the active magnetic bearings due to unbalance are also investigated and are found to show the same behaviour as the displacement except for large rotational speed.

Rotor and housing lateral displacements caused by vehicle acceleration reach up to $30, \mu \mathrm{m}$ which is almost three times larger than the non-gimballed case. However, it is still only $15 \%$ of the maximum allowable lateral displacement of $200 \mu \mathrm{m}$. The housing is swinging in the gimbal up to $28^{\circ}$. The rotor displacement and AMB forces are found to be significantly depending on the ratio of mass moment of inertia of housing and rotor and on the length from gimbal pivot point to rotor-housing center of mass.

The investigated flywheel system demonstrates how the gimbal effectively removes the large gyroscopic forces but introduces other problems such as a housing swinging up to $28^{\circ}$ and almost three times larger rotor displacements. The examples also demonstrate how selected physical design parameters are significantly affecting the forces and the dynamics thus proving how the model is relevant for proper system design.

In a future work it would be relevant to look at a parametric study of all the input parameters instead of just the few selected here. Especially, the control of the active magnetic bearings could be optimised using the developed multi-physical model.

Furthermore, a thorough experimental validation of the global model is to be presented in a future work. The validation will encompass comparisons of experimental and theoretical results for the sub-models: AMB dynamics, PMB forces, and multibody dynamics as well as comparisons of experiments and theory when combining the sub-models into a global model.

\section{Acknowledgements}

The authors gratefully acknowledge the economic support from the Innovation Fund Denmark Grant and the valuable input from Maersk Drilling and WattsUp Power.

\section{APPENDIX}

\section{Appendix A. Input Parameters}

All simulation cases discussed in the report other than the examples use the same parameters as example 1 except for $\phi(t)$ and $\Omega$ which are zero. 


\begin{tabular}{|c|c|c|c|c|}
\hline & Unit & Example 1 & Example 2 & Example 3 \\
\hline$\Omega_{\text {earth }}$ & $\frac{\mathrm{rad}}{\mathrm{s}}$ & 0 & 0 & 0 \\
\hline$\delta$ & [rad] & 0 & 0 & 0 \\
\hline$\psi(t)$ & {$[\mathrm{rad}]$} & 0 & 0 & 0 \\
\hline$\theta(t)$ & [rad] & 0 & 0 & 0 \\
\hline$\phi(t)$ & {$[\mathrm{rad}]$} & $0.79 \sin (0.52 t)$ & 0 & 0 \\
\hline$\ddot{x}_{1}$ & $\mathrm{~m} / \mathrm{s}^{2}$ & 0 & 0 & 2.455 \\
\hline$\ddot{y}_{1}$ & $\mathrm{~m} / \mathrm{s}^{2}$ & 0 & 0 & 0 \\
\hline$\ddot{z}_{1}$ & $\mathrm{~m} / \mathrm{s}^{2}$ & 0 & 0 & 0 \\
\hline$x_{3-4}$ & {$[\mathrm{~m}]$} & 0 & 0 & 0 \\
\hline$y_{3-4}$ & {$[\mathrm{~m}]$} & 0 & 0 & 0 \\
\hline$z_{3-4}$ & {$[\mathrm{~m}]$} & 0.413 & 0.413 & 0.413 \\
\hline$l$ & {$[\mathrm{~m}]$} & 0.015 & 0.015 & $0-0.3$ \\
\hline$a$ & {$[\mathrm{~m}]$} & 0.038 & 0.038 & 0.038 \\
\hline$b$ & {$[\mathrm{~m}]$} & 0.038 & 0.038 & 0.038 \\
\hline$c$ & {$[\mathrm{~m}]$} & 0.08 & 0.08 & 0.08 \\
\hline$d$ & {$[\mathrm{~m}]$} & 0.08 & 0.08 & 0.08 \\
\hline$e$ & {$[\mathrm{~m}]$} & 0.152 & 0.152 & 0.152 \\
\hline G & {$[\mathrm{m}]$} & 0.158 & 0.158 & 0.158 \\
\hline$h$ & {$[\mathrm{~m}]$} & 0.158 & 0.158 & 0.158 \\
\hline$\mu_{y_{1}}$ & {$\left[\mathrm{~m}^{2} \mathrm{~N} / \mathrm{s}\right]$} & 0.014 & 0.014 & 0.014 \\
\hline$\mu_{x_{3}}$ & {$\left[\mathrm{~m}^{2} \mathrm{~N} / \mathrm{s}\right]$} & 0.007 & 0.007 & 0.007 \\
\hline$m_{r}$ & {$[\mathrm{~kg}]$} & 3.2 & 3.2 & 3.2 \\
\hline$m_{h}$ & {$[\mathrm{~kg}]$} & 3.2 & 3.2 & 3.2 \\
\hline$m_{g}$ & {$[\mathrm{~kg}]$} & 1.6 & 1.6 & 1.6 \\
\hline$I_{r_{p}}$ & {$\left[\mathrm{~m}^{2} \mathrm{~g}\right]$} & 3.8 (nominal) & 3.8 & 3.8 \\
\hline$I_{h_{p}}$ & {$\left[\mathrm{~m}^{2} \mathrm{~g}\right]$} & 7.6 & 7.6 & 7.6 \\
\hline$I_{g_{p}}$ & {$\left[\mathrm{~m}^{2} \mathrm{~g}\right]$} & 3.8 & 3.8 & 3.8 \\
\hline$I_{r_{t}}$ & {$\left[\mathrm{~m}^{2} \mathrm{~g}\right]$} & 22 & 22 & 22 \\
\hline$I_{h_{t}}$ & {$\left[\mathrm{~m}^{2} \mathrm{~g}\right]$} & 22 & $2.2-44$ & $2.2-44$ \\
\hline$I_{g_{t}}$ & {$\left[\mathrm{~m}^{2} \mathrm{~g}\right]$} & 2.2 & $0.22-22$ & 2.2 \\
\hline$\tau_{m / g}$ & {$[\mathrm{~N} / \mathrm{m}]$} & 0 & 0 & 0 \\
\hline$u$ & {$[\mathrm{~g} \cdot \mathrm{mm}]$} & 0 & 6.4 & 0 \\
\hline$l_{u}$ & $\mathrm{~m}$ & 0 & 0.15 & 0 \\
\hline$g$ & {$\left[\mathrm{~m} / \mathrm{s}^{2}\right]$} & 9.82 & 9.82 & 9.82 \\
\hline$M$ & {$[\mathrm{kA} / \mathrm{m}]$} & 750 & 750 & 750 \\
\hline$w$ & {$[\mathrm{~mm}]$} & 2.3 & 2.3 & 2.3 \\
\hline$n_{z}$ & {$[-]$} & 10 & 10 & 10 \\
\hline$n_{r}$ & {$[-]$} & 3 & 3 & 3 \\
\hline$r$ & {$[\mathrm{~mm}]$} & $21.3,24.3,27.3$ & $21.3,24.3,27.3$ & $21.3,24.3,27.3$ \\
\hline$k_{r}$ & {$[\mathrm{kN} / \mathrm{m}]$} & -20 & -20 & -20 \\
\hline$k_{z}$ & {$[\mathrm{kN} / \mathrm{m}]$} & 40 & 40 & 40 \\
\hline
\end{tabular}




\begin{tabular}{|c|c|c|c|c|}
\hline & Unit & Example 1 & Example 2 & Example 3 \\
\hline$z_{0}$ & {$[\mathrm{~m}]$} & 0 & 0 & 0 \\
\hline$h_{0}$ & {$[\mathrm{~mm}]$} & 2.3 & 2.3 & 2.3 \\
\hline$\mu_{0}$ & {$[\mathrm{H} / \mathrm{m}]$} & $4 \pi \cdot 10^{-7}$ & $4 \pi \cdot 10^{-7}$ & $4 \pi \cdot 10^{-7}$ \\
\hline$A$ & {$\left[\mathrm{~m}^{2}\right]$} & 0.000364 & 0.000364 & 0.000364 \\
\hline$n$ & {$[-]$} & 350 & 350 & 350 \\
\hline$i_{\text {bias }}$ & {$[\mathrm{A}]$} & 0.6 & 0.6 & 0.6 \\
\hline$x_{0}$ & {$[\mathrm{~mm}]$} & 0.45 & 0.45 & 0.45 \\
\hline$K_{c}$ & {$[\mathrm{~V} / \mathrm{A}]$} & 150 & 150 & 150 \\
\hline$K_{p}$ & {$[\mathrm{~A} / \mathrm{m}]$} & 3000 & 3000 & 3000 \\
\hline$T_{d}$ & {$[\mathrm{~ms}]$} & 1.2 & 1.2 & 1.2 \\
\hline$K_{i}$ & {$[1 / \mathrm{s}]$} & 3.33 & 3.33 & 3.33 \\
\hline \multicolumn{5}{|c|}{ Initial values of state variables: } \\
\hline$\Omega$ & {$[\mathrm{RPM}]$} & 30000 & $0-30000$ & 0 \\
\hline All o & r states & 0 & 0 & 0 \\
\hline
\end{tabular}

\section{Appendix B. Linearised AMB Equations}

The AMB forces Eq. (34)-(37) are linearised around linearisation point $i_{0}=i_{\text {bias }}$ and a centred rotor with air gap $x_{0}$ yielding:

$$
\begin{gathered}
f_{x_{5}, \text { lin }}=\cos (\pi / 8)\left(k_{i} i_{x_{A}}+k_{s} x_{b_{A}}\right) \\
f_{y_{5}, \text { lin }}=\cos (\pi / 8)\left(k_{i} i_{y_{A}}+k_{s} y_{b_{A}}\right) \\
f_{x_{6}, \text { lin }}=\cos (\pi / 8)\left(k_{i} i_{x_{B}}+k_{s} x_{b_{B}}\right) \\
f_{y_{6}, \text { lin }}=\cos (\pi / 8)\left(k_{i} i_{y_{B}}+k_{s} y_{b_{B}}\right)
\end{gathered}
$$

where the force-current factor is

$$
k_{i}=\mu_{0} n^{2} A \frac{i_{0}}{x_{0}^{2}}
$$

and the force-displacement factor is

$$
k_{s}=k_{i} \frac{i_{0}}{x_{0}}
$$

The AMB coil currents 40 are linearised around the same linearisation point as the forces. In total, eight coils are present and thus eight equations govern the currents. However after linearisation, the coils can be paired in $\left(i^{+}, i^{-}\right)$-pairs such that e.g.

$$
\begin{aligned}
& i_{x_{A}}^{+}=i_{\mathrm{bias}}+i_{x_{A}} \\
& i_{x_{A}}^{-}=i_{\mathrm{bias}}-i_{x_{A}}
\end{aligned}
$$


where $i_{x_{A}}(t)$ is the control current. This means that the linearised coil equations become:

$$
\begin{array}{r}
R i_{x_{A}}+L \dot{i}_{x_{A}}+k_{i} \dot{x}_{b_{A}}=-K_{c}\left(K_{p}\left(T_{d} \dot{x}_{\mathrm{se}_{A}}+x_{\mathrm{se}_{A}}+K_{i} e_{x_{\mathrm{se}_{A}}}\right)+i_{x_{A}}\right) \\
R i_{y_{A}}+L \dot{i}_{y_{A}}+k_{i} \dot{y}_{b_{A}}=-K_{c}\left(K_{p}\left(T_{d} \dot{y}_{\mathrm{se}_{A}}+y_{\mathrm{se}_{A}}+K_{i} e_{y_{\mathrm{se}_{A}}}\right)+i_{y_{A}}\right) \\
R i_{x_{B}}+L \dot{i}_{x_{B}}+k_{i} \dot{x}_{b_{B}}=-K_{c}\left(K_{p}\left(T_{d} \dot{x}_{\mathrm{se}_{B}}+x_{\mathrm{se}_{B}}+K_{i} e_{x_{\mathrm{se}_{B}}}\right)+i_{x_{B}}\right) \\
R i_{y_{B}}+L \dot{i}_{y_{B}}+k_{i} \dot{y}_{b_{B}}=-K_{c}\left(K_{p}\left(T_{d} \dot{y}_{\mathrm{se}_{B}}+y_{\mathrm{se}_{B}}+K_{i} e_{y_{\mathrm{se}_{B}}}\right)+i_{y_{B}}\right)
\end{array}
$$

where the inductance is

$$
L=\frac{\mu n^{2} A}{2 x_{0}}
$$

and the motion induced voltage coefficient $k_{u}$ is equal to $k_{i}$ in this ideal case of no eddy-currents, flux leakage, or magnetic hysteresis.

\section{Appendix C. System of Equations}

Here, we present a simplified version of the system of equations obtained following the procedure in Sec. 2. The simplifications are:

$$
\begin{array}{rl}
\ddot{y_{1}}=0 & \ddot{z_{1}}=0 \\
\mathbf{r}_{3-4}=\mathbf{0} & z=0 \\
\theta=0 & \psi=0 \\
\phi=0 & \Omega_{0}=0 \\
\delta=0 &
\end{array}
$$

The system uses these simplifications in order to fit into a normal size page and in order for a human rather than a computer to be able to read it. It should serve well for benchmarking the results from examples 2 and 3 as it includes all significant dynamics needed in these cases. The equations Eq. 18)-23) (except for rotor force equilibrium in axial direction), Eq. (B.9)-B.12, and Eq. 411) are set up as

$$
\mathbf{A}(\mathbf{t}) \mathbf{x}(\mathbf{t})=\mathbf{b}(\mathbf{t})
$$

and solved for the unknowns

$$
\begin{array}{r}
\mathbf{x}=\left\{\ddot{x} \ddot{y} \ddot{\eta} \ddot{\zeta} \dot{\Omega} \dot{i}_{x_{A}} \dot{i}_{y_{A}} \dot{i}_{x_{B}} \dot{i}_{y_{B}} \dot{e}_{x_{\mathrm{se}_{A}}} \dot{e}_{y_{\mathrm{se}_{A}}} \dot{e}_{x_{\mathrm{se}_{B}}} \dot{e}_{y_{\mathrm{se}_{B}}} \ldots\right. \\
\left.\ldots f_{x_{1}} f_{y_{1}} f_{z_{1}} f_{x_{2}} f_{z_{2}} f_{x_{3}} f_{y_{3}} f_{z_{3}} f_{y_{4}} f_{z_{4}} \ddot{\alpha} \ddot{\beta}\right\}^{\mathrm{T}} .
\end{array}
$$

In the following, $\cos ()$ and $\sin ()$ are abbreviated $c()$ and $s()$. The system matrix $\mathbf{A}$ and the right-hand side b are: 


$\mathbf{A}=\left[\begin{array}{ccccccccccccccccccc}m_{f} & 0 & 0 & 0 & 0 & 0 & 0 & 0 & 0 & 0 & 0 & 0 & 0 & 0 & 0 & 0 & 0 & 0 & 0 \\ 0 & m_{f} & 0 & 0 & 0 & 0 & 0 & 0 & 0 & 0 & 0 & 0 & 0 & 0 & 0 & 0 & 0 & 0 & 0 \\ 0 & 0 & I_{r_{t}} & 0 & 0 & 0 & 0 & 0 & 0 & 0 & 0 & 0 & 0 & 0 & 0 & 0 & 0 & 0 & 0 \\ 0 & 0 & 0 & I_{r_{t}} c(\eta) & 0 & 0 & 0 & 0 & 0 & 0 & 0 & 0 & 0 & 0 & 0 & 0 & 0 & 0 & 0 \\ 0 & 0 & 0 & -I_{f_{p}} s(\eta) & I_{f_{p}} & 0 & 0 & 0 & 0 & 0 & 0 & 0 & 0 & 0 & 0 & 0 & 0 & 0 & 0 \\ 0 & 0 & 0 & 0 & 0 & L & 0 & 0 & 0 & 0 & 0 & 0 & 0 & 0 & 0 & 0 & 0 & 0 & 0 \\ 0 & 0 & 0 & 0 & 0 & 0 & L & 0 & 0 & 0 & 0 & 0 & 0 & 0 & 0 & 0 & 0 & 0 & 0 \\ 0 & 0 & 0 & 0 & 0 & 0 & 0 & L & 0 & 0 & 0 & 0 & 0 & 0 & 0 & 0 & 0 & 0 & 0 \\ 0 & 0 & 0 & 0 & 0 & 0 & 0 & 0 & L & 0 & 0 & 0 & 0 & 0 & 0 & 0 & 0 & 0 & 0 \\ 0 & 0 & 0 & 0 & 0 & 0 & 0 & 0 & 0 & 1 & 0 & 0 & 0 & 0 & 0 & 0 & 0 & 0 & 0 \\ 0 & 0 & 0 & 0 & 0 & 0 & 0 & 0 & 0 & 0 & 1 & 0 & 0 & 0 & 0 & 0 & 0 & 0 & 0 \\ 0 & 0 & 0 & 0 & 0 & 0 & 0 & 0 & 0 & 0 & 0 & 1 & 0 & 0 & 0 & 0 & 0 & 0 & 0 \\ 0 & 0 & 0 & 0 & 0 & 0 & 0 & 0 & 0 & 0 & 0 & 0 & 1 & 0 & 0 & 0 & 0 & 0 & 0 \\ 0 & 0 & 0 & 0 & 0 & 0 & 0 & 0 & 0 & 0 & 0 & 0 & 0 & -1 & 0 & 0 & -1 & 0 & -1 \\ 0 & 0 & 0 & 0 & 0 & 0 & 0 & 0 & 0 & 0 & 0 & 0 & 0 & 0 & -1 & 0 & 0 & 0 & 0 \\ 0 & 0 & 0 & 0 & 0 & 0 & 0 & 0 & 0 & 0 & 0 & 0 & 0 & 0 & 0 & -1 & 0 & -1 & 0 \\ 0 & 0 & 0 & 0 & 0 & 0 & 0 & 0 & 0 & 0 & 0 & 0 & 0 & 0 & 0 & G & 0 & -G & 0 \\ 0 & 0 & 0 & 0 & 0 & 0 & 0 & 0 & 0 & 0 & 0 & 0 & 0 & 0 & 0 & 0 & 0 & 0 & 0 \\ 0 & 0 & 0 & 0 & 0 & 0 & 0 & 0 & 0 & 0 & 0 & 0 & 0 & -G & 0 & 0 & G & 0 & 0 \\ 0 & 0 & 0 & 0 & 0 & 0 & 0 & 0 & 0 & 0 & 0 & 0 & 0 & 0 & 0 & 0 & 0 & 0 & 1 \\ 0 & 0 & 0 & 0 & 0 & 0 & 0 & 0 & 0 & 0 & 0 & 0 & 0 & 0 & 0 & 0 & 0 & 0 & 0 \\ 0 & 0 & 0 & 0 & 0 & 0 & 0 & 0 & 0 & 0 & 0 & 0 & 0 & 0 & 0 & 0 & 0 & 0 & 0 \\ 0 & 0 & 0 & 0 & 0 & 0 & 0 & 0 & 0 & 0 & 0 & 0 & 0 & 0 & 0 & 0 & 0 & 0 & 0 \\ 0 & 0 & 0 & 0 & 0 & 0 & 0 & 0 & 0 & 0 & 0 & 0 & 0 & 0 & 0 & 0 & 0 & 0 & 0 \\ 0 & 0 & 0 & 0 & 0 & 0 & 0 & 0 & 0 & 0 & 0 & 0 & 0 & 0 & 0 & 0 & 0 & 0 & 0\end{array}\right]$

0
0
0
0
0
0
0
0
0
0
0
0
0
0
-1
0
0
0
$h$
0
$c(\beta)$
$-s(\beta)$
0
$-h s(\beta)$
$-h c(\beta)$

$\begin{array}{ccc}0 & 0 & 0 \\ 0 & 0 & 0 \\ 0 & 0 & 0 \\ 0 & 0 & 0 \\ 0 & 0 & 0 \\ 0 & 0 & 0 \\ 0 & 0 & 0 \\ 0 & 0 & 0 \\ 0 & 0 & 0 \\ 0 & 0 & 0 \\ 0 & 0 & 0 \\ 0 & 0 & 0 \\ 0 & 0 & 0 \\ 0 & 0 & 0 \\ 0 & -1 & 0 \\ -1 & 0 & -1 \\ 0 & 0 & 0 \\ -h & 0 & h \\ 0 & -h & 0 \\ 0 & 0 & 0 \\ s(\beta) & c(\beta) & s(\beta) \\ c(\beta) & -s(\beta) & c(\beta) \\ 0 & 0 & 0 \\ h c(\beta) & h s(\beta) & -h c(\beta) \\ -h s(\beta) & h c(\beta) & h s(\beta)\end{array}$

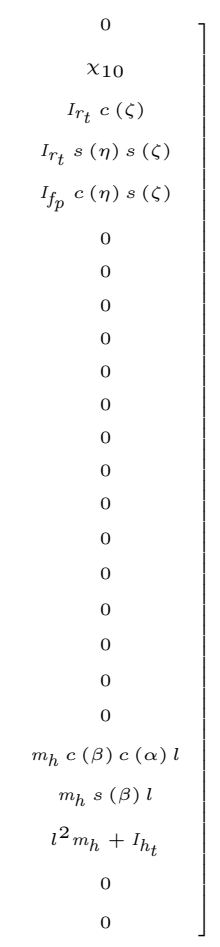

$\chi_{1}=m_{f}\left(-l(c(\alpha)+1)(c(\beta))^{2}+(s(\beta) c(\alpha) y+s(\beta) y-s(\alpha) x) c(\beta)+l\right)$ $\chi_{2}=m_{f}\left(-s(\beta) x c(\alpha)+s(\alpha)\left(l c(\beta) s(\beta)+y(c(\beta))^{2}-y\right)\right)$ $\chi_{3}=I_{r_{t}} s(\zeta) s(\beta)$ $\chi_{4}=I_{r_{t}}(c(\eta) c(\beta)-s(\eta) c(\zeta) s(\beta))$ $\chi_{5}=I_{r_{p}}(-s(\eta) c(\beta)-c(\eta) c(\zeta) s(\beta))$ $\chi_{6}=-m_{h}\left(-1+(c(\alpha)+1)(c(\beta))^{2}\right) l$ $\chi_{7}=m_{h} s(\beta) c(\beta) s(\alpha) l$ $\chi_{8}=m_{h} l s(\alpha)(c(\beta))^{2}$ $\chi_{9}=c(\beta)\left(l^{2} m_{h}+I_{h_{t}}\right)$

$\chi_{10}=m_{f}((l c(\beta)-s(\beta) y) c(\alpha)+s(\alpha) x)$ 
$-I_{r_{p}}(-\dot{\eta} c(\eta)(c(\beta) \dot{\alpha}+\dot{\zeta})+s(\eta) \dot{\beta} s(\beta) \dot{\alpha}-\dot{\eta} s(\eta)(s(\zeta) \dot{\beta}-c(\zeta) s(\beta) \dot{\alpha})+c(\eta)(-c(\zeta) \dot{\beta} c(\beta) \dot{\alpha}+\dot{\zeta} s(\zeta) s(\beta) \dot{\alpha}+\dot{\zeta} c(\zeta) \dot{\beta}))$

$$
K_{c}\left(-K_{p}\left(T_{d}(c \dot{\zeta}+\dot{x})+e_{x_{s e}}+x+\zeta c\right)-i_{x_{A}}\right)-R x A i_{x_{A}}-k_{i}(a \dot{\zeta}+\dot{x})
$$

$K_{c}\left(-K_{p}\left(T_{d}(-c \dot{\eta}+\dot{y})+e_{y_{s e}}+y-\eta c\right)-i_{y_{A}}\right)-R y A i_{y_{A}}-k_{i}(-a \dot{\eta}+\dot{y})$

$K_{C}\left(-K_{p}\left(T_{d}(-d \dot{\zeta}+\dot{x})+e_{x_{s e}}+x-\zeta d\right)-i_{x_{B}}\right)-R x B i_{x_{B}}-k_{i}(-b \dot{\zeta}+\dot{x})$

$K_{c}\left(-K_{p}\left(T_{d}(d \dot{\eta}+\dot{y})+e_{y_{s e}}+y+\eta d\right)-i_{y_{B}}\right)-R y B i_{y_{B}}-k_{i}(b \dot{\eta}+\dot{y})$

$K_{i}(\zeta c+x)$

$K_{i}(-\eta c+y)$

$K_{i}(-\zeta d+x)$

$K_{i}(\eta d+y)$

$s(\alpha) m_{g} g-m_{g} c(\alpha) \ddot{x}_{1}$

0

$-m_{g} s(\alpha) \ddot{x}_{1}-c(\alpha) m_{g} g$

$\mu_{x_{3}} \dot{\beta}$

$-\mu_{y_{1}} \dot{\alpha}$

0

$\kappa_{5}$

$\kappa 7$

$\frac{\kappa 8}{c(\eta) c(\zeta)}-\left(c(\beta)\left(l^{2} m_{h}-I_{h_{p}}+I_{h_{t}}\right) \dot{\alpha}^{2}+l s(\alpha) m_{h} \ddot{x}_{1}\right) s(\beta)$

$\kappa_{9}$

$$
\frac{\kappa_{10}}{c(\eta) c(\zeta)}+I_{h_{p}} \dot{\beta} c(\beta) \dot{\alpha}
$$

$k_{1}=\Omega^{2} c(\Omega t) u+k_{i} i_{x_{A}}+k_{s}(a \zeta+x)+k_{i} i_{x_{B}}+k_{s}(-b \zeta+x)+c(\zeta) k_{r}(-e \zeta+x)+s(\eta) s(\zeta) k_{r}(e \eta+y)+s(\alpha) m_{f} g$

$-m_{f}\left(c(\beta)\left(l s(\alpha)(c(\alpha)+1)(c(\beta))^{2}+\left(-x(c(\alpha))^{2}+s(\alpha)(-s(\beta) y+l) c(\alpha)-s(\beta) s(\alpha) y+x\right) c(\beta)-s(\beta) c(\alpha) s(\alpha) y-x(c(\alpha))^{2}-l s(\alpha)\right) \dot{\alpha}^{2}\right.$

$+\left(\left(y(c(\alpha)+1)^{2}(c(\beta))^{3}+(c(\alpha)+1)(l s(\beta) c(\alpha)+l s(\beta)+y)(c(\beta))^{2}+\left(-(c(\alpha))^{2} y+((s(\alpha) x+l) s(\beta)-y) c(\alpha)+(s(\alpha) x+l) s(\beta)-y\right) c(\beta)-y c(\alpha)-s(\beta)(-s(\alpha) x+l)\right) \beta\right.$

$\left.+2 c(\beta)(s(\beta) c(\alpha) \dot{y}+s(\beta) \dot{y}-s(\alpha) \dot{x})) \dot{\alpha}+c(\alpha) \ddot{x}_{1}\right)$

395

${ }^{2}=\Omega^{2} s(\Omega t) u+k_{i} i_{y_{A}}+k_{\mathrm{s}}(-a \eta+y)+k_{i} i_{\eta_{B}}+k_{\mathrm{s}}(b \eta+y)+c(\eta) k_{r}(e \eta+y)-s(\beta) c(\alpha) m_{f} g-m_{f}\left(\left((c(\beta)+1)\left(l c(\beta) s(\beta)+y(c(\beta))^{2}-y\right)(c(\alpha))^{2}\right.\right.$

$\left.+(c(\beta)+1)\left(y(c(\beta))^{2}+(l s(\beta)-y) c(\beta)-s(\beta)(-s(\alpha) x+l)\right) c(\alpha)-l c(\beta) s(\beta)-y(c(\beta))^{2}+y\right) \dot{\alpha}^{2}+\left(\left(-x(c(\beta)+1)(c(\beta)-2)(c(\alpha))^{2}+\left(l(c(\beta))^{3} s(\alpha)-s(\alpha)(s(\beta) y+l)(c(\beta))^{2}\right.\right.\right.$ $\left.+((s(\beta) y-2 l) s(\alpha)-x) c(\beta)+2 s(\beta) s(\alpha) y) c(\alpha)+l(c(\beta))^{3} s(\alpha)+(-s(\beta) s(\alpha) y+x)(c(\beta))^{2}+(-l s(\alpha)-x) c(\beta)+l s(\alpha)-x\right) \dot{\beta}$

$\left.\left.+2(c(\beta))^{2} s(\alpha) \dot{y}-2 s(\beta) \dot{x} c(\alpha)-2 s(\alpha) \dot{y}\right) \dot{\alpha}-c(\alpha)\left(\left(l c(\beta) s(\beta)+y(c(\beta))^{2}-y\right) c(\alpha)+l c(\beta) s(\beta)+y(c(\beta))^{2}+s(\beta) s(\alpha) x\right) \dot{\beta}^{2}+(-2 s(\beta) c(\alpha) \dot{y}+2 s(\alpha) \dot{x}) \dot{\beta}+s(\beta) s(\alpha) \ddot{x}_{1}\right)$

$400 \quad \kappa_{3}=-l_{u} \Omega^{2} s(\Omega t) u-\frac{a\left(s(\eta) s(\zeta)\left(k_{i} i_{x_{A}}+k_{s}(a \zeta+x)\right)+c(\eta)\left(k_{i} i_{y_{A}}+k_{s}(-a \eta+y)\right)\right)}{c(\eta) c(\zeta)}+\frac{b\left(s(\eta) s(\zeta)\left(k_{i} i_{x_{B}}+k_{s}(-b \zeta+x)\right)+c(\eta)\left(k_{i} i_{y_{B}}+k_{s}(b \eta+y)\right)\right)}{c(\eta) c(\zeta)}+\frac{e k_{r}(e \eta+y)}{c(\eta) c(\zeta)}-I_{f_{t}}(s(\zeta) \dot{\beta} c(\beta) \dot{\alpha}+\dot{\zeta} c(\zeta) s(\beta) \dot{\alpha}-\zeta s(\zeta) \dot{\beta})-$ $(c(\eta)(c(\beta) \dot{\alpha}+\dot{\zeta})+s(\eta)(s(\zeta) \dot{\beta}-c(\zeta) s(\beta) \dot{\alpha})) I_{r_{p}}(-s(\eta)(c(\beta) \dot{\alpha}+\dot{\zeta})+c(\eta)(s(\zeta) \dot{\beta}-c(\zeta) s(\beta) \dot{\alpha})+\Omega)+(-s(\eta)(c(\beta) \dot{\alpha}+\dot{\zeta})+c(\eta)(s(\zeta) \dot{\beta}$ $-c(\zeta) s(\beta) \dot{\alpha})) I_{f_{t}}(c(\eta)(c(\beta) \dot{\alpha}+\dot{\zeta})+s(\eta)(s(\zeta) \dot{\beta}-c(\zeta) s(\beta) \dot{\alpha}))$

$k_{4}=l_{u} \Omega^{2} c(\Omega t) u+\frac{a\left(k_{i} i_{x_{A}}+k_{s}(a \zeta+x)\right)}{c(\eta)}-\frac{b\left(k_{i} i_{x_{B}}+k_{s}(-b \zeta+x)\right)}{c(\eta)}-\frac{k_{r}(-e \zeta+x) e}{c(\eta) c(\zeta)}-I_{f t}(-\dot{\eta} s(\eta)(c(\beta) \dot{\alpha}+\dot{\zeta})-c(\eta) \dot{\beta} s(\beta) \dot{\alpha}+\dot{\eta} c(\eta)(s(\zeta) \dot{\beta}-c(\zeta) s(\beta) \dot{\alpha})$

$405+s(\eta)(-c(\zeta) \dot{\beta} c(\beta) \dot{\alpha}+\dot{\zeta} s(\zeta) s(\beta) \dot{\alpha}+\dot{\zeta} c(\zeta) \dot{\beta}))+(c(\zeta) \dot{\beta}+s(\zeta) s(\beta) \dot{\alpha}+\dot{\eta}) I_{r_{p}}(-s(\eta)(c(\beta) \dot{\alpha}+\dot{\zeta})+c(\eta)(s(\zeta) \dot{\beta}-c(\zeta) s(\beta) \dot{\alpha})+\Omega)$

$-(-s(\eta)(c(\beta) \dot{\alpha}+\dot{\zeta})+c(\eta)(s(\zeta) \dot{\beta}-c(\zeta) s(\beta) \dot{\alpha})) I_{f_{t}}(c(\zeta) \dot{\beta}+s(\zeta) s(\beta) \dot{\alpha}+\dot{\eta})$ 
$\kappa_{5}=-k_{i} i_{x_{A}}-k_{s}(a \zeta+x)-k_{i} i_{x_{B}}-k_{s}(-b \zeta+x)-c(\zeta) k_{r}(-e \zeta+x)-s(\eta) s(\zeta) k_{r}(e \eta+y)+s(\alpha) m_{h} g$

410

酒 $\left((c(\beta)+1) s(\alpha)((c(\alpha)+1) c(\beta)-1) c(\beta) l \dot{\alpha}^{2}+s(\beta)\left(-1+(c(\alpha)+1)^{2}(c(\beta))^{2}+(c(\alpha)+1) c(\beta)\right) \dot{\beta} l \dot{\alpha}+c(\alpha) \ddot{x}_{1}\right)$

$\kappa_{6}=-k_{i} i_{y_{A}}-k_{s}(-a \eta+y)-k_{i} i_{y_{B}}-k_{s}(b \eta+y)-c(\eta) k_{r}(e \eta+y)-s(\beta) c(\alpha) m_{h} g-m_{h}\left(s(\beta)\left(\left((c(\alpha))^{2}+c(\alpha)\right)(c(\beta))^{2}+\left((c(\alpha))^{2}-1\right) c(\beta)-c(\alpha)\right) l \dot{\alpha}^{2}\right.$ $\left.+\left(1+(c(\alpha)+1)(c(\beta))^{3}-(c(\beta))^{2} c(\alpha)+(-2 c(\alpha)-1) c(\beta)\right) s(\alpha) \dot{\beta} l \dot{\alpha}-s(\beta)\left(l c(\beta) c(\alpha)(c(\alpha)+1) \dot{\beta}^{2}-s(\alpha) \ddot{x}_{1}\right)\right)$

$\kappa_{7}=s(\zeta) k_{r}(-e \zeta+x)-s(\eta) c(\zeta) k_{r}(e \eta+y)-c(\beta) c(\alpha) m_{h} g-m_{h}\left(c(\beta)\left(\left((c(\alpha))^{2}+c(\alpha)\right)(c(\beta))^{2}+\left((c(\alpha))^{2}-1\right) c(\beta)-c(\alpha)\right) l \dot{\alpha}^{2}\right.$

$\left.-s(\beta) s(\alpha)(1+(c(\alpha)+1) c(\beta)) c(\beta) \dot{\beta} l \dot{\alpha}+\left(-1+(c(\alpha)+1)(c(\beta))^{2}\right) l \dot{\beta}^{2}+c(\beta) s(\alpha) \ddot{x}_{1}\right)$

$\kappa_{8}=-\mu_{x_{3}} \dot{\beta} c(\eta) c(\zeta)-l(e \eta+y) c(\zeta) k_{r}(c(\eta))^{2}-c(\zeta)\left(s(\eta)(e \eta+y) y k_{r} c(\zeta)-y k_{r}(-e \zeta+x) s(\zeta)+l s(\beta) c(\alpha) m_{h} g+\left(a(-l+a) k_{s}+k_{s} b(l+b)\right) \eta-y(-l+a) k_{s}+y(l+b) k_{s}\right.$ $\left.-a i_{y_{A}} k_{i}+b i_{y_{B}} k_{i}+l\left(k_{i} i_{y_{A}}+k_{i} i_{y_{B}}\right)\right) c(\eta)+((-e \eta-y) c(\zeta)+s(\eta) s(\zeta)(-e \zeta+x)) k_{r} e$

420

$\kappa_{9}=\frac{(x(e \eta+y) s(\eta)+(-e \zeta+x) l) k_{r}(c(\zeta))^{2}+\left(-k_{r}(-(e \eta+y) l s(\eta)+x(-e \zeta+x)) s(\zeta)-l s(\alpha) m_{h} g+\left(\left(a k_{s}-b k_{s}\right) l-a^{2} k_{s}-b^{2} k_{s}\right) \zeta+\left(k_{i} i_{x_{A}}+k_{i} i_{x_{B}}+2 k_{s} x\right) l+\left(-a k_{s}+b k_{s}\right) x-a i_{x_{A}} k_{i}+i_{x_{B}} k_{i} b\right) c(\zeta)+k_{r}(-e \zeta+x) e}{c(\zeta)}$ $+2 \dot{\beta}\left(l^{2} m_{h}-I_{h_{p}} / 2+I_{h_{t}}\right) s(\beta) \dot{\alpha}+m_{h} l c(\alpha) \ddot{x}_{1}$

$\kappa_{10}=-(e \eta+y) c(\zeta) k_{r} x(c(\eta))^{2}+\left((-e \zeta+x) y k_{r}(c(\zeta))^{2}+\left((e \eta+y) y k_{r} s(\eta) s(\zeta)+\left(a k_{s}-b k_{s}\right) x \eta+y\left(a k_{s}-b k_{s}\right) \zeta+\left(k_{i} i_{x_{A}}+k_{i} i_{x_{B}}\right) y-x\left(k_{i} i_{y_{A}}+k_{i} i_{y_{B}}\right)\right) c(\zeta)\right.$ $\left.+\left(\left(a^{2} k_{s}+b^{2} k_{s}\right) \eta+\left(-a k_{s}+b k_{s}\right) y-a i_{y_{A}} k_{i}+b i_{y_{B}} k_{i}\right) s(\zeta)\right) c(\eta)+(-e \zeta+x) k_{r} s(\eta) e c(\zeta)+(e \eta+y) k_{r} e s(\zeta)-\left(\left(a^{2} k_{s}+b^{2} k_{s}\right) \zeta+\left(a k_{s}-b k_{s}\right) x+a i_{x_{A}} k_{i}-i_{x_{B}} k_{i} b\right) s(\eta)$ 


\section{Appendix D. Comsol Solver Settings}

For physical parameters used, see Appendix A, example 1. COMSOL settings:

Physics: Magnetic fields, no currents (mfnc), stationary, 3D. Magnetic flux conservation is used to impose magnetic moment of $M$ in magnet rings. Magnetic flux conservation is also used in surrounding air. Relative permeability of both air and magnets are 1. Mesh: Tetrahedral, maximum element size: $1 \mathrm{~mm}$, minimum element size: $0.01 \mathrm{~mm}$, maximum element growth rate: 1.3, curvature factor: 0.2 , resolution of narrow regions: 1. Only half of rotor and stator is simulated. Boundaries of domain is set minimum $10 \mathrm{~mm}$ away from any magnet (except for boundary at cross-section) and magnetic insulation conditions are imposed on all boundaries.

\section{References}

[1] T. Dever, Presentation: Development of a high specific energy flywheel module, and studies to quantify its mission applications and benefits, available online: http://ntrs.nasa.gov/archive/nasa/casi.ntrs.nasa.gov/20150009522.pdf [Accessed Sep. 5th 2016] (2013).

口 [2] I. E. Agency, Technology roadmap, electric and plug-in hybrid electric vehicles, available online: https://www.iea.org/ publications/freepublications/publication/EV_PHEV_Roadmap.pdf [Accessed Jan. 23th 2018] (2009).

[3] T. Higuchi, L. Hawkins, Keynote Speeches ISMB 2016, 15th International Symposium on Magnetic Bearings (2016) 1-28.

[4] A. Power, Overview of flywheel technology, available online: http://www . activepower.com/cleansource-750HD/[Accessed Sep. 12th 2016] (2016).

[5] B. Power, $20 \mathrm{MW}$ flywheel energy storage plant, available online: http://www.sandia.gov/ess/docs/pr_conferences/ 2014/Thursday/Session7/02_Areseneaux_Jim_20MW_Flywheel_Energy_Storage_Plant_140918.pdf [Accessed Sep. 12th 2016].

[6] W. R. Canders, H. May, J. Hoffmann, P. Hoffmann, F. Hinrichsen, I. Koch, D. Röstermundt, Flywheel mass energy storage with hts bearing - development status, available online: http://www.eurosolar.org/new/pdfs_neu/electric/IRES2006_ Canders.pdf [Accessed Sep. 12th 2016] (2006).

[7] C. M. Reid, T. B. Miller, M. A. Hoberecht, P. L. Loyselle, L. M. Taylor, S. C. Farmer, R. H. Jansen, History of Electrochemical and Energy Storage Technology Development at NASA Glenn Research Center, Journal of Aerospace Engineering 26 (2) (2013) 361-371. doi:10.1061/(ASCE) AS.1943-5525.0000323 URL http://ascelibrary.org/doi/10.1061/\{\%\}28ASCE\{\%\}29AS.1943-5525.0000323

[8] M. Hedlund, J. Lundin, J. de Santiago, J. Abrahamsson, H. Bernhoff, Flywheel Energy Storage for Automotive Applications, Energies 8 (10) (2015) 10636-10663. doi:10.3390/en81010636 URL http://www.mdpi.com/1996-1073/8/10/10636/

[9] M. Amiryar, K. Pullen, A Review of Flywheel Energy Storage System Technologies and Their Applications, Applied Sciences 7 (3) (2017) 286. doi:10.3390/app7030286 URL http://www .mdpi . com/2076-3417/7/3/286

[10] A. A. K. Arani, H. Karami, G. B. Gharehpetian, M. S. A. Hejazi, Review of Flywheel Energy Storage Systems structures and applications in power systems and microgrids Renewable and Sustainable Energy Reviews 69 (September 2016) (2017) 9-18. doi:10.1016/j.rser.2016.11.166. URL http://dx.doi.org/10.1016/j.rser.2016.11.166 
[11] S. M. G. Mousavi, F. Faraji, A. Majazi, K. Al-haddad, A comprehensive review of Flywheel Energy Storage System technology, Renewable and Sustainable Energy Reviews 67 (2017) 477-490. doi:10.1016/j.rser.2016.09.060 URL http://dx.doi .org/10.1016/j.rser.2016.09.060

[12] J. Tang, B. Xiang, Y. Zhang, Dynamic characteristics of the rotor in a magnetically suspended control moment gyroscope with active magnetic bearing and passive magnetic bearing, ISA Transactions 53 (4) (2014) 1357-1365. doi:10.1016/j. isatra.2014.03.009

[13] J. Fang, S. Zheng, B. Han, AMB Vibration Control for Structural Resonance of Double-Gimbal Control Moment Gyro With High-Speed Magnetically Suspended Rotor, IEEE/ASME TRANSACTIONS ON MECHATRONICS 18 (1) (2013) $32-43$.

[14] P. Kascak, Demonstration of attitude control and bus regulation with flywheels, Conference Record - IAS Annual Meeting (IEEE Industry Applications Society) 3 (2004) 2018 - 2029.

[15] P. Tsiotras, Satellite attitude control and power tracking with energy/momentum wheels, Journal of Guidance Control and Dynamics 24 (1) (2001) 23 - 34.

[16] W. H. Power, Flywheel energy storage, available online: http://www.ukintpress-conferences.com/uploads/SPKPMW13R/ d1_s1_p2_ian_foley.pdf [Accessed Sep. 5th 2016] (2013).

[17] J. Wheals, Rail hybrid using flywheel, available online: http://www.banekonference.dk/sites/default/files/slides/ 11/1130_NEW_Ricardo\%20Rail\%20Hybrid\%20Using\%20Flywheel.pdf [Accessed Aug. 28th 2017], Den Danske Banekonference 2016, Tivoli Congress Centre, Copenhagen (2016).

[18] C. Jarroux, J. Mahfoud, R. Dufour, B. Defoy, T. Alban, On the dynamics of rotating machinery supported by AMB during base motion, 15th International Symposium on Magnetic Bearings (2016) 1-8.

[19] S. Y. Yoon, Z. Lin, T. Dimond, P. E. Allaire, Control of Active Magnetic Bearing systems on non-static foundations, in: 2011 9th IEEE International Conference on Control and Automation (ICCA), IEEE, 2011, pp. 556-561. doi:10.1109/ ICCA.2011.6137957 URL http://ieeexplore.ieee.org/lpdocs/epic03/wrapper.htm?arnumber=6137957

[20] T. Dimond, P. Allaire, S. Mushi, Z. Lin, S. Y. Yoon, Modal tilt/translate control and stability of a rigid rotor with gyroscopics on active magnetic bearings, International Journal of Rotating Machinery (2012) 1-10 doi:10.1155/2012/ 567670 .

[21] B. Murphy, D. A. Bresie, J. H. Beno, Bearing Loads in a Vehicular Flywheel Battery, SAE Special Publications, Electric and Hybrid Vehicle Design Studies, Proceedings of the 1997 International Congress and Expositiondoi:10.4271/970213

[22] L. Hawkins, B. Murphy, R. Hayes, J. Zierer, Shock and vibration testing of an AMB supported energy storage flywheel, JSME International Journal 46 (2) (2003) 429-435.

[23] M. M. Flynn, J. J. Zierer, R. C. Thompson, Performance testing of a vehicular flywheel energy system, SAE Technical Papersdoi:10.4271/2005-01-0809

[24] B. Rachmanto, K. Nonami, K. Kuriyama, H. Shimazaki, T. Kagamiishi, T. Moriya, A study on amb flywheel powered electric vehicle, Journal of System Design and Dynamics 3 (4) (2009) 659-670. doi:10.1299/jsdd.3.659

[25] F. Shimizu, K. Nonami, Benchmark and Verification of Control Algorithm for Flywheel with Active Magnetic Bearing on Electric Vehicle and Proposal of New SAC Algorithm ( $\epsilon 1$ modification and bias variable $\Gamma$ p approach ), ISMB14 (2014) 313-318.

[26] C. Huynh, P. Mcmullen, A. Filatov, S. Imani, H. A. Toliyat, S. Talebi, Flywheel energy storage system for naval applications, Proceedings of the ASME Turbo Expo 5 PART A (2006) 25 - 33.

[27] J. McGroarty, J. Schmeller, R. Hockney, M. Polimeno, Flywheel energy storage system for electric start and an all-electric ship, 2005 IEEE Electric Ship Technologies Symposium 2005 (0704) (2005) 400-406. doi:10.1109/ESTS.2005.1524706

[28] W. Schiehlen, Technische Dynamik [Technical dynamics], Teubner Studienbuche, 1986. 
[29] F. Pfeiffer, Einführung in die Dynamik [Introduction to dynamics], Teubner Studienbuche, 1988.

[30] H. Bremer, F. Pfeiffer, Elastische Mehrkorpersysteme [Elastic multi-body systems], Teubner Studienbuche, 1992.

[31] I. F. Santos, Dinâmica De Sistemas Mecanicos [Dynamics of mechanical systems], Ed. Makron Books, São Paulo, Brazil, 2001.

[32] E. Estupinan, I. F. Santos, Modelling hermetic compressors using different constraint equations to accommodate multibody dynamics and hydrodynamic lubrication, Brazilian Society of Mechanical Sciences and Engineering. Journal 31 (1) (2009) $35-46$.

[33] S. Lahriri, I. F. Santos, Theoretical modelling, analysis and validation of the shaft motion and dynamic forces during rotor-stator contact, Journal of Sound and Vibration 332 (24) (2013) 6359-6376. doi:10.1016/j.jsv.2013.07.008.

[34] W. Breinl, Entwurf eines unempfindlichen tragregelsystems für ein magnetschwebefahrzeug [design of a robust force control system for a levitated vehicle], PhD thesis, 34 (1980) 134.

[35] M. Hedlund, J. Abrahamsson, J. Lundin, H. Bernhoff, Passive Axial Thrust Bearing for a Flywheel Energy Storage System (2013) 1-13.

[36] C. Toh, S. Chen, Design, Modeling and Control of Magnetic Bearings for a Ring-Type Flywheel Energy Storage System, Energies 9 (12) (2016) 1051. doi:10.3390/en9121051. URL http://www .mdpi .com/1996-1073/9/12/1051

[37] N. Tanase, A. M. Morega, A permanent magnet bearing for flywheel energy storage systems - numerical modeling, International Conference on Applied and Theoretical Electricity (2016) 1-5.

[38] G. G. Sotelo, E. Rodriguez, F. S. Costa, J. G. Oliveira, J. de Santiago, R. M. S., Tests with a hybrid bearing for a flywheel energy storage system, Superconductor science and technology 29 (2016) 1-10.

[39] S. I. Bekinal, S. Jana, Generalized Three-Dimensional Mathematical Models for Force and Stiffness in Axially, Radially, and Perpendicularly Magnetized Passive Magnetic Bearings With "n" Number of Ring Pairs, Journal of Tribology 138 (3) (2016) 031105. doi:10.1115/1.4032668 URL http://tribology . asmedigitalcollection. asme.org/article.aspx?doi=10.1115/1.4032668

[40] M. Siebert, B. Ebihara, R. Jansen, R. L. Fusaro, W. Morales, A. Kascak, A. Kenny, A Passive Magnetic Bearing Flywheel, NASA TM-2002-211159.

[41] M. Baatz, M. Hyrenbach, Method of calculating the magnetic flux density and forces in contact-free magnetic bearings, European Transactions on Electrical Power 1 (4) (1991) 195-199. doi:10.1002/etep.4450010404

[42] A. Olejnik, K. Falkowski, Passive magnetic bearings at the rotary application, Mechanisms and Machine Science 21 (2015) 1477-1487. doi:10.1007/978-3-319-06590-8_121

[43] D. J. Griffiths, Introduction to electrodynamics, Prentice-Hall, 1981.

[44] S. B. Andersen, S. Enemark, I. F. Santos, Dynamics and stability of rigid rotors levitated by passive cylinder-magnet bearings and driven/supported axially by pointwise contact clutch Journal of Sound and Vibration 332 (25) (2013) 66376658. doi:10.1016/j.jsv.2013.07.006 URL http://dx.doi.org/10.1016/j.jsv.2013.07.006

[45] D. Vischer, Sensorlose und spannungsgesteuerte magnetlager [sensorless and voltage controlled magnetic bearings], Federal Institute of Technology (ETH), Zurich, Switzerland, PhD thesis, No. 8665 (1988) 120.

[46] D. Meeker, Finite element method magnetics, version 4.2, user's manual, available online: http://www.femm.info/ Archives/doc/manual42.pdf [Accessed Nov. 28th 2017] (October 25, 2015).

[47] D. Laier, Nichtlinearitäten magnetgelagerter rotorsysteme [nonlinear magnetic bearing rotor system], PhD thesis, Technische Universität Darmstadt (1998) 86.

[48] Magnetic Bearings: Theory, Design, and Application To Rotating Machinery, Springer Berlin Heidelberg, 2009.

[49] Cogent Power, Datasheet for Hi-Lite NO30, available online: https://cogent-power.com/cms-data/downloads/Hi-Lite_ 
N030.pdf [Accessed May. 18th 2018] (2016).

550

[50] COMSOL, Comsol multiphysics, available online: https://www.comsol.com/comsol-multiphysics [Accessed Nov. 28th 2017].

[51] COMSOL, Permanent magnet, application id: 78.

[52] R. Hockney, M. Polimeno, G. Robinson, Flywheel Energy Storage in Support of Naval Integrated Power Systems Contract N00024-09-C-2407.

[53] Iso standard 1940-1. mechanical vibration — balance quality requirements for rotors in a constant (rigid) state — part 1: Specification and verification of balance tolerances.

[54] B. G. Johnson, K. Misovec, R. Hockney, Synchronous response modelling and control of an annular momentum control device, Contractor Report 4166. 